\title{
The 10d uplift of the GPPZ solution
}

\author{
Michela Petrini, ${ }^{a}$ Henning Samtleben, ${ }^{b}$ Stanislav Schmidt ${ }^{c}$ and Kostas Skenderis ${ }^{c}$ \\ a Sorbonne Université, CNRS, Laboratoire de Physique Théorique et Hautes Énergies, \\ F-75005 Paris, France \\ ${ }^{b}$ Univ Lyon, Ens de Lyon, Univ Claude Bernard, CNRS, Laboratoire de Physique, \\ F-69342 Lyon, France \\ ${ }^{c}$ STAG Research Centre and Mathematical Sciences, University of Southampton, \\ Southampton, SO17 1BJ, U.K. \\ E-mail: Petrini@lpthe.jussieu.fr, Henning.Samtleben@ens-lyon.fr, \\ S.Schmidt@soton.ac.uk, K.Skenderis@soton.ac.uk
}

ABSTRACT: We present the uplift of the GPPZ solution of the five-dimensional maximal supergravity to ten dimensions. The five dimensional solution involves two real scalar fields, with one of them encoding holographically the (norm of the complex) supersymmetric $\mathcal{N}=1$ mass deformation and the other the real part of the gaugino condensate. We embed this solution in a consistent truncation of $D=5$ maximal supergravity which involves two complex scalars dual to the complex mass deformations and the complex gaugino condensate, and a $\mathrm{U}(1)$ gauge field dual to the $\mathrm{U}(1)_{R}$ current, and uplift it to ten dimensions. The ten dimensional solution is completely explicit, with all fields given in terms of elementary functions. The metric and the axion-dilaton agree with those of a partial uplift of the GPPZ flow by Pilch and Warner. We analyze the asymptotics and the singularity structure of the ten dimensional solution. The uplifted solution is singular, but the singularity is milder than that of the five dimensional solution, and there is conformal frame in which the metric is only singular at one point of $S^{5}$. We compare the asymptotics of the $10 d$ solution with that of the Polchinski-Strassler and Freedman-Minahan solutions, and find agreement with Freedman-Minahan and disagreement with Polchinski-Strassler. In particular, we infer that while the Polchinski-Strassler $10 d$ fields satisfy the correct boundary conditions, they do not solve the field equations near the boundary.

KEYwORDS: AdS-CFT Correspondence, Gauge-gravity correspondence

ARXiv EPRINT: 1805.01919 


\section{Contents}

1 Introduction and summary of results 2

2 The GPPZ flow $\quad 6$

3 Uplift of the GPPZ solution $\quad 10$

$\begin{array}{lll}3.1 & \text { Four-scalar truncation of } D=5 \text { supergravity } & 10\end{array}$

3.2 Uplift of the 4-scalar truncation: metric and dilaton/axion 12

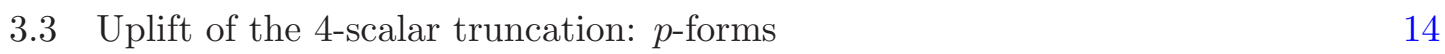

$\begin{array}{lll}3.4 & \text { Five-form field strength and self-duality equations } & 15\end{array}$

$\begin{array}{lll}3.5 & \text { Dual 6-forms } & 17\end{array}$

$\begin{array}{lll}3.6 & \text { Einstein equations } & 18\end{array}$

$\begin{array}{lll}4 & \text { UV asymptotics of the uplifted solution } & 18\end{array}$

5 Singularity $\quad 20$

5.1 The $\lambda<1$ case 21

5.2 The $\lambda=1$ case 24

5.3 Different frames 25

6 Conclusions and outlook $\quad 26$

A IIB uplift of $D=5$ maximal supergravity 28

B Parametrization of the scalar matrix $\quad 30$

C Uplift in Pilch-Warner coordinates

$\begin{array}{ll}\text { D } S^{5} \text { spherical harmonics with } \mathrm{SO}(3)_{\text {diag }} \text { symmetry } & 36\end{array}$

E UV asymptotics of the GPPZ uplift

$\begin{array}{lll}\text { F One field truncations } & 41\end{array}$

F.1 The case $m(y)=0 \quad 41$

$\begin{array}{lll}\text { F.2 The case } \sigma(y)=0 & 43\end{array}$ 


\section{Introduction and summary of results}

Since the early days of the AdS/CFT correspondence many efforts have been devoted to constructing gravity duals of $\mathcal{N}=1$ gauge theories in four dimensions. While we have now a very good understanding of the duality for superconformal $\mathcal{N}=1$ theories, the same is not true for non-conformal ones. The two best known $\mathcal{N}=1$ solutions, the Maldacena-Nunez [1] and the Klebanov-Strassler [2] ones, are dual to gauge theories with unconventional UV completions, namely higher-dimensional theories or theories with an infinite number of degrees of freedom. A natural theory to be studied holographically is $\mathcal{N}=1^{*}$. This is obtained as a deformation of $\mathcal{N}=4$ Super Yang Mills and, according to the AdS/CFT dictionary, its supergravity dual should correspond to a deformation of $A d S_{5} \times S^{5}$. However the issue of finding such solution is still not settled.

The $\mathcal{N}=1^{*}$ theory is obtained by adding a mass term for the three chiral superfields of $\mathcal{N}=4$ Super Yang Mills. This amounts to adding to the $\mathcal{N}=4$ superpotential the term

$$
\delta \mathcal{W}=m_{i j} \operatorname{tr}\left(\Phi_{i} \Phi_{j}\right)
$$

which reduces the supersymmetry to $\mathcal{N}=1$ and breaks explicitly the R-symmetry. At energies lower than the mass scale the chiral matter multiplets decouple, the U(1) Rsymmetry is recovered and the theory flows to pure $\mathcal{N}=1$ Super Yang Mills. $\mathcal{N}=1$ Super Yang Mills confines and has a mass gap, with $N$ different vacua associated with the gaugino condensates.

Far from the decoupling limit, the $\mathcal{N}=1^{*}$ theory has a rich structure of vacua as described in [3-5]. Some vacua are characterised by a mass gap, which can be due to a Higgs mechanism or to confinement, while some others contain massless photons and hence have no mass gap. At the classical level the vacua are parameterised by the $N$ dimensional representations of $\mathrm{SU}(2)$, as one can see from the $\mathrm{F}$-term equation ${ }^{1}$

$$
\left[\Phi_{i}, \Phi_{j}\right]=-\frac{m}{\sqrt{2}} \epsilon_{i j k} \Phi_{k}
$$

The $N$-dimensional irreducible representation of $\mathrm{SU}(2)$ corresponds to the Higgs vacuum. The gauge group is completely broken and there is a mass gap already at the classical level. As the theory is weakly coupled at all energy scales, the semiclassical analysis holds also at the quantum level and there is exactly one vacuum. The opposite case, corresponding to $N$ copies of the trivial representation, is the $\mathrm{SU}(N)$ confining vacuum. All the vevs of the scalars are zero and the gauge group is unbroken. At the quantum level the theory confines in the IR and the classical vacuum splits into $N$ vacua parameterised by the gaugino condensate $\langle\lambda \lambda\rangle$. Since the R-symmetry is explicitly broken already in the UV, the different vacua are not related by a discrete R-symmetry as in pure $\mathcal{N}=1$ Super Yang Mills, and are not isomorphic. In each vacuum the superpotential takes different values and the domain walls connecting the vacua have different tension. This is the vacuum that survives in the decoupling limit.

\footnotetext{
${ }^{1}$ We consider the case where the mass term is diagonal. By rescaling the superfields $\Phi_{i}$ one can always write the mass term as $m_{i j}=m \delta_{i j}$.
} 
Other confining vacua appear when the fields $\Phi_{i}$ consist of blocks that are all in the same representation of dimension $p$. The residual gauge group is $\mathrm{SU}(p)$. At the quantum level the theory confines and there are $p$ vacua parameterised by the gaugino condensates. If, on the contrary, the $\Phi_{i}$ split in blocks of the different dimensions, the vacua will generically have U(1) factors and, hence, massless photons. These vacua have no mass gap and are called Coulomb vacua.

A first attempt to determine the gravity dual of the $\mathcal{N}=1^{*}$ theory is the so called GPPZ solution [6] in $\mathcal{N}=8 \mathrm{SO}(6)$ gauged supergravity in five-dimensions [7, 8]. This is a consistent truncations of type IIB supergravity on $A d S_{5} \times S^{5}[9]$ that keeps only the lightest modes of the Kaluza-Klein reduction on $S^{5}$ [10]. On the gauge theory side these modes correspond to the relevant operators in $\mathcal{N}=4$ Super Yang Mills and thus contain the mass deformations. By imposing that the masses of three chiral superfields are the same it is possible to consistently truncate the five-dimensional supergravity to only two scalars, $m$ and $\sigma$, and to find an analytic solution of the five-dimensional equations of motion. The asymptotic behaviour of the two scalars confirm that $m$ and $\sigma$ can be identified with a mass term for the matter superfields and the gaugino condensate, respectively.

The solution has a naked singularity. Nevertheless it is possible to perform several computations on the solution and obtain sensible results that seem to confirm its interpretation as the dual of $\mathcal{N}=1^{*} .^{2}$ It can be easily checked that the theory admits a mass gap and a discrete spectrum of glueballs [13]. Moreover, the holographic computation of 2-point functions along the flow gave results consistent with interpretation of the solution as a deformation of $\mathcal{N}=4 \mathrm{SYM}$, including subtle issues regarding the analytic structure of the correlators $[14,15]$. The GPPZ solution admits two truncations, with $\sigma=0$ and with $m=0$, respectively. For the $m=0$ solution the spectrum of light scalars has been computed in [16] and appears to be insensitive to the IR singularity. On the other hand, [17] found a massless state in the spectrum of theory, which may point towards the interpretation of the solution as being dual to a Coulomb vacuum. Even if the solution has many qualitative features consistent with being dual of one of the confining vacua of $\mathcal{N}=1^{*}$, due to the naked singularity, its physical interpretation is still not completely clear. Sometimes singularities are resolved when a solution is uplifted in higher dimensions [18]. For example, the solutions describing the Coulomb Branch of $\mathcal{N}=4 \mathrm{SYM}$ have a naked singularity from the $5 d$ perspective but they are non-singular from the $10 d$ perspective [19]. Therefore, the first step towards assessing the validity of the GPPZ solution is to uplift it to 10 dimensions and this is the topic of this paper.

\footnotetext{
${ }^{2}$ In [11] it was argued that there cannot be a supergravity description of the confining vacua of $\mathcal{N}=1^{*}$ theory. This claim was based on the structure of the vacuum expectation values at strong coupling, which was obtained using an alleged modular symmetry of the chiral sector of the theory in each vacuum. The existence of this symmetry relied on a contribution of the Konishi anomaly to the $\mathbf{1 0}$ and $\overline{\mathbf{1 0}}$, which is now known not to be correct. In more detail, equation (2.4) of [11] contains a quantum contribution to the 10. The operator in the $\mathbf{1 0}$ however is a supersymmetric operators and as such it cannot mix with the non-supersymmetric Konishi operator (and this has been checked explicitly to 2-loop order in [12].). This invalidates equation (4.9) in [11] and consequently the derivation of the vevs at strong coupling. We thank Ofer Aharony for a discussion about this point.
} 
The general uplift of $\mathcal{N}=8 \mathrm{SO}(6)$ gauge supergravity to type IIB was constructed in [9]. Here our starting point is a 4 -scalar truncation of the five-dimensional supergravity. As described above $\mathcal{N}=1^{*}$ is a deformation of the $\mathcal{N}=4$ SYM superpotential by an Fterm, which is a complex operator. The gaugino bi-linear is also a complex operator and, therefore, their sources are complex, making a total of 4 real scalars in the dual supergravity. In addition, consistency of the $5 d$ supergravity theory requires that we include a $\mathrm{U}(1)$ gauge field. ${ }^{3}$ Altogether this gives an $\mathrm{SO}(3)$ invariant truncation of $D=5$ supergravity that involves the metric, 2 complex scalars, $\underline{m}=m e^{i \varphi}$ and $\underline{\sigma}=\sigma e^{i \omega}$ and a $\mathrm{U}(1)$ gauge field. ${ }^{4}$ The GPPZ solution lives in a further (consistent) truncation of this theory that keeps the norms $m$ and $\sigma$ and truncates the angles $\varphi, \omega$ and the gauge field.

The uplift of the 4-scalar sector has a number of interesting features. It turns out that the angles of the complex scalars are completely accounted for by a combination of an $\mathrm{SO}(2)$ rotation of the coordinates of $S^{5}$ and an $\mathrm{SO}(2)_{\text {IIB }}$ rotation of the $\mathrm{SL}(2)_{\text {IIB }}$ symmetry of IIB supergravity. To be more precise, let $\left(u^{i}, v^{i}\right)$ be coordinates on $\mathbb{R}^{6}$ such that each triplet parameterises an $\mathbb{R}^{3}$. The $S^{5}$ is then described by $u^{2}+v^{2}=1$ and the $\mathrm{S} O(3)$ symmetry acts by a simultaneous $\mathrm{S} O(3)$ rotation of $u^{i}$ and $v^{i}$. The first $\mathrm{S} O(2)$ rotates the $u$ 's into $v$ 's and geometrises the $\mathrm{U}(1)_{R}$ action of the dual QFT. The $\mathrm{S} O(2)_{\text {IIB }}$ corresponds to the bonus $\mathrm{U}(1)$ symmetry of $\mathcal{N}=4 \mathrm{SYM}[25]$ and it is the $\mathrm{U}(1)_{Y}$ group discussed in [26] in the context of the $S^{5}$ compactification of IIB supergravity. It is also worth mentioning that the periodicity of the angles $\varphi$ and $\omega$ in $D=5$ maps in ten dimensions to the invariance under the combined operation of exchanging of $u^{i}$ with $v^{i}$ and performing an S-duality transformation.

Applying the uplift formulae to the GPPZ flow we find an explicit ten-dimensional solution of type IIB, with all metric coefficients, the dilaton-axion and the $p$-forms given in terms of elementary functions. All $p$-forms are turned on in the solution, in particular both the NSNS and RR 2-form potentials (as mentioned in the previous paragraph, the solution is invariant under $S$ duality, which exchanges the two potentials, followed by an exchange of the coordinates $u^{i}$ and $v^{i}$ ). The ten-dimensional metric and the axion-dilaton agree exactly with those of the partial uplift of Pilch-Warner [20]. We checked using Mathematica [27] that the type IIB equations are satisfied.

The solution is asymptotically $A d S_{5} \times S^{5}$ and the leading correction is due to the 2 -form potentials, as expected based on the spectrum of linear perturbations [10] and the AdS/CFT dictionary. We also give the first few sub-leading terms in the asymptotic expansion in order to compare with other solutions that appeared in the literature. The first few sub-leading terms are uniquely fixed by the boundary conditions [28] and by working out the asymptotic expansion to sufficiently high order one can extract (in principle) the vevs of all gauge invariant operators using the method of Kaluza-Klein holography [29]. We will report on this computation elsewhere.

\footnotetext{
${ }^{3} \mathrm{In} \mathcal{N}=1$ language, the $\mathrm{SU}(4)_{R}$ R-symmetry of $\mathcal{N}=4 \mathrm{SYM}$ decomposes as $\mathrm{SU}(3) \times \mathrm{U}(1)_{R}$. The gauge field that we keep is dual to the $\mathrm{U}(1)_{R}$.

${ }^{4}$ This 4-scalar sector has been considered earlier in $[20,21]$. One can further truncate this theory by setting either $\underline{m}=0$ or $\underline{\sigma}=0$. The $\underline{m}=0$ truncation and its uplift to 10 dimensions has been discussed in [22-24]. We discuss both cases in appendix F.
} 
The solution is still singular in ten dimensions but the divergence is milder than that of the five-dimensional solution. In five dimensions the entire spacetime metric goes to zero as we approach the singularity. In ten dimensions the singularity structure depends on the size of the deformation parameter relative to the gaugino condensate, which is quantified by a parameter $\lambda$. It was argued in [6] that the singularity is acceptable provided $\lambda \leq 1$. The singularity structure is different depending on whether $\lambda<1$ or $\lambda=1$. The $\lambda=1$ is similar to the $5 d$ solution, with a singularity both in the non-compact directions and the compact spherical part. When $\lambda<1$ the non-compact part of the metric is now nonsingular and there is only a singularity in the compact spherical part, a singularity which was called "ring singularity" in [20]. More precisely, following [20] we view $S^{5}$ as an $\mathbb{R P}^{3}$ fibered over a disc. The singularity is located at the edge of the disc. In [20] it was argued that the singularity is associated with 7-branes but we find no evidence for 7-branes in the near-singularity structure of the metric.

It turns out that the singularity structure also depends on the choice of frame: one can rescale the metric with appropriate powers of the scalars fields in the solution. While in the usual frames, the Einstein and string frame, the singularity structure is similar, there are also frames where the ten-dimensional metric is only singular at one point, a point at the edge of the disc. It would be interesting to understand the physics behind this observation.

It is possible that the supergravity approximation is not sufficient to describe duals of $\mathcal{N}=1^{*}$ theories and that some stringy mechanism is needed to resolve the singularity. In [5] Polchinski and Strassler suggested that the five-dimensional singularity is resolved by D3-branes polarised via Myers' effect [30] into five-branes with world-volume $\mathbb{R}^{4} \times S^{2}$, where $S^{2}$ is an equator of $S^{5}$ and $\mathbb{R}^{4}$ is a slice of $A d S_{5}$ at fixed radius. The construction is motivated by the observation that the F-term condition (1.2) tell us that the branes are non-commutatively expanded into two spheres. ${ }^{5}$ Consider, for instance, the Higgs vacuum. The Dirac-Born-Infeld-Wess-Zumino action for the D3 branes becomes [30]

$$
S \sim \mu_{3} \int C_{4}+\mu_{3}\left(2 \pi \alpha^{\prime}\right)^{2} \int F_{0123 i j k}\left[x^{i}, x^{j}\right] x^{k},
$$

and we see that the expanded D3-branes have an additional electric coupling to the RR 6form and are therefore equivalent to a single D5-brane with $N$ units of D3-brane charge and zero net D5-brane charge. Then the Higgs vacuum is identified with a single D5-brane. Sduality transformations can be used to construct the backgrounds dual to the other vacua. The confining vacua are identified with single NS-branes, while the others are superpositions of D5 and NS5 branes. This interpretation also agrees with the AdS/CFT dictionary since the fermionic mass term in (1.1) corresponds the lowest KK-mode in the expansion of the complex two-form field of type IIB and this latter is exactly the potential needed to polarise D3-branes into 5-branes. However, only the asymptotic solutions near the boundary and the branes were given [5] and the full supergravity solutions corresponding to the various brane configurations are not known. Comparing this solution with the uplifted GPPZ solution, we find that the boundary conditions (the sources) agree, but the subleading terms in the near-boundary expansion disagree. Since the first few sub-leading terms are

\footnotetext{
${ }^{5}$ The scalar components of the superfields $\Phi_{i}$ are the transverse coordinates of the branes $\left(x^{i}=2 \pi \alpha^{\prime} \phi_{i}\right)$.
} 
uniquely fixed in terms of the sources, we conclude that the Polchinski-Strassler $10 d$ field do not satisfy the IIB equations near the boundary.

The finite temperature physics of $\mathcal{N}=1^{*}$ theory was studied holographically by Freedman and Minahan [31] by deforming the solution of a black D3-brane by adding the complex combination of the RR and NS 3-forms dual to the mass terms. The solution is obtained perturbatively up to second order in the deformation parameter. We can use $T=0$ limit of Freedman-Minahan solution as a perturbative check of our solution in the UV, and we find that the two solutions are indeed in agreement.

The paper is organised as follows. In the next section we review the five-dimensional GPPZ solution. In section 3 we present the uplift of the 4 scalar sector of the $D=$ 5 supergravity and of the GPPZ solution. In section 4 we discuss the near-boundary asymptotics and in section 5 we analyse the singularity structure of the ten-dimensional solution. We conclude with an outlook in section 6 . The paper contains also a number of appendices. In appendix A we summarise the uplift formulas from [9] and in appendix B we present the explicit parameterisation of the scalar $\mathrm{E}_{6(6)}$ matrix in the 4-scalar truncation of $D=5$ maximal supergravity. In appendix $\mathrm{C}$ we present the ten-dimensional solution in Pilch-Warner coordinates and in appendix D we list the $\mathrm{SO}(3)$ invariant spherical harmonics that we use in the main text. Finally, in appendix F we present the uplift of the two onescalar truncations of the GPPZ solution.

\section{The GPPZ flow}

The GPPZ flow [6] is a solution of the five-dimensional $\mathcal{N}=8 \mathrm{SO}(6)$ gauged supergravity [7, 8]. The field content of the theory can be organised in representations of the $\mathrm{SO}(6) \times \mathrm{SL}(2)$ subgroup of $E_{6(6)}$. It consists of 15 massless vectors fields in the adjoint of $\mathrm{SO}(6), 12$ topologically massive two-forms transforming in the $(\mathbf{6}, \mathbf{2})$ of $\mathrm{SO}(6) \times \mathrm{SL}(2)$ and 42 scalars parameterising an $E_{6(6)} / \mathrm{USp}(8)$ coset space and transforming as

$$
42=\mathbf{2 0}_{(0)}^{\prime}+10_{(-2)}+\overline{\mathbf{1 0}}_{(2)}+\mathbf{1}_{(4)}+\mathbf{1}_{(-4)},
$$

where the subscripts are the charges under the $\mathrm{U}(1)_{Y}$ subgroup of $\mathrm{SL}(2)$ [26]. The masses of these scalar are $m^{2}=-4$ (for the $\mathbf{2 0}$ ), $m^{2}=-3$ (for the $\mathbf{1 0}$ and $\overline{\mathbf{1 0}}$ ) and $m^{2}=0$ (for the two 1s).

According to the AdS/CFT dictionary, the 42 scalars are dual to relevant and marginal operators $^{6}$ of $\mathcal{N}=4 \mathrm{SYM} . \mathcal{N}=4 \mathrm{SYM}$ contains six scalars $\phi_{i}$ and four fermions $\lambda_{a}$ transforming in the $\mathbf{6}$ and $\mathbf{4}$ of SU(4), respectively. Then the scalars in the $\mathbf{2 0}$ correspond to scalar bilinears $\operatorname{Tr} \phi_{(i} \phi_{j}$ (-traces) of conformal dimension $\Delta=2$, the scalars in the $\mathbf{1 0}$ are massive deformations with $\Delta=3$, schematically

$$
\left.Q^{2} \operatorname{Tr} \phi_{(i} \phi_{j}\right) \operatorname{Tr}\left(\lambda_{a} \lambda_{b}+\phi^{3}\right)
$$

and the scalars in the $\mathbf{1}$ are the gauge-coupling deformations.

\footnotetext{
${ }^{6}$ Recall that the mass of an AdS scalar is mapped to the conformal dimension of the dual field theory operator by $\Delta=2+\sqrt{4+m^{2}}$.
} 
We are interested in massive deformations of $\mathcal{N}=4 \mathrm{SU}(N)$ SYM that break supersymmetry to $\mathcal{N}=1$. In $\mathcal{N}=1$ notation the six scalar fields $\phi_{i}$ are arranged in three complex scalars, which together with three of the four fermions form three chiral superfields $\Phi_{i}, i=1,2,3$, while the remaining fermion sits in the vector multiplet. Of the full $\mathrm{SO}(6)$ R-symmetry only a $\mathrm{U}(1)_{R} \times \mathrm{SU}(3)_{R}$ subgroup is manifest, under which the vector superfield is neutral and the three chiral superfields transform in the $\mathbf{3}_{2 / 3}$.

To identify the relevant $5 d$ scalars we need to describe the mass deformation in more detail. In $\mathcal{N}=1$ language the mass deformation modifies the superpotential of the theory by the addition of the term

$$
\delta \mathcal{W}=m_{i j} \operatorname{tr}\left(\Phi_{i} \Phi_{j}\right)
$$

that only involves three of the four $\mathcal{N}=4$ fermions. Thus we need to decompose the $\mathrm{SU}(4) \sim \mathrm{SO}(6)$ representations into $\mathrm{SU}(3) \times \mathrm{U}(1)$ and single out the gaugino. The fundamental of $\mathrm{SU}(4)$ splits as

$$
4 \rightarrow 3+1
$$

and thus the four fermions $\lambda_{a}$ in the $\mathbf{4}$ of SU(4) split into the $\mathbf{3}$ corresponding to the three fermions in the chiral multiplets $\Phi_{i}$ and the $\mathbf{1}$ which is the gaugino $\lambda$. The fermionic mass term in the $\mathbf{1 0}$ then decomposes as

$$
10 \rightarrow 1+3+6
$$

and we identify the $\mathbf{6}$ with the mass deformation, while the scalar in the $\mathbf{1}$ corresponds to a gaugino condensate. Integrating out the auxiliary fields we find that $\mathcal{N}=4 \mathrm{SYM}$ the generic mass deformation also involves, on the gravity side, part of the scalars in the $\mathbf{2 0}^{\prime},{ }^{7}$ unless the three fermion masses are taken to be equal (the details will be reported in [32]). If the masses are equal, the part corresponding to the $\mathbf{2 \mathbf { 0 } ^ { \prime }}$ does not appear, and there is a residual $\mathrm{SO}(3)$ symmetry that allows us to keep only two holomorphic scalars, $\underline{\sigma} \in \mathbf{1}$ and $\underline{m} \in \mathbf{6}$, while setting all the remaining fields consistently to zero. These two fields are dual to the operators 8

$$
\mathcal{O}_{3}=\sum_{i=1}^{3} \operatorname{tr}\left(\lambda_{i} \lambda_{i}\right), \quad \mathcal{O}_{4}=\operatorname{tr}\left(\lambda_{4} \lambda_{4}\right) .
$$

Similarly, we get two anti-holomorphic scalars, $\underline{\bar{\sigma}}, \underline{\bar{m}}$ (the complex conjugates of $\underline{\sigma}$ and $\underline{m}$ ) from the $\overline{\mathbf{1 0}}$. This is as expected, since $\underline{m}$ and $\underline{\sigma}$ are dual to chiral operators.

In AdS/CFT the QFT generating functional of correlation functions becomes the onshell value of the bulk action. Since $\underline{m}$ and $\underline{\sigma}$ couple to complex operators, the generating functional will only contain the modulus of $\underline{m}$ and $\underline{\sigma}$. Indeed, in $\mathcal{N}=4 \mathrm{SYM}$, $\left\langle\mathcal{O}_{3} \mathcal{O}_{3}\right\rangle=\left\langle\overline{\mathcal{O}}_{3} \overline{\mathcal{O}}_{3}\right\rangle=0$ but $\left\langle\mathcal{O}_{3} \overline{\mathcal{O}}_{3}\right\rangle \neq 0$ and the same with $\mathcal{O}_{3} \rightarrow \mathcal{O}_{4}$, which means the generating function will depend on $|\underline{m}|^{2},|\underline{\sigma}|^{2}$ but not on $\underline{m}^{2}, \underline{\bar{m}}^{2}, \underline{\sigma}^{2}, \underline{\bar{\sigma}}^{2}$, and similarly for

\footnotetext{
${ }^{7}$ The mass deformation also involves the Konishi operator, which however decouples in the supergravity limit.

${ }^{8}$ These operators are obtained from $\mathbf{2 0}$ by acting with two supercharges and they contain also a part proportional to $\phi^{3}$ that we suppress here.
} 
the contributions coming from higher point functions. Indeed, we will see in the next section that there is consistent truncation of the bulk supergravity to the moduli $m$ and $\sigma$ of $\underline{m}$ and $\underline{\sigma}$,

$$
\underline{m}=m e^{i \varphi}, \quad \underline{\sigma}=\sigma e^{i \omega} .
$$

We are thus lead to look for $5 d$ solutions of the form

$$
d s^{2}=d y^{2}+e^{2 \phi(y)} d x^{\mu} d x_{\mu}
$$

with $\mu=0, \ldots, 3$ and non-trivial profile for the real fields $m(y)$ and $\sigma(y)$. The radial coordinate $y$ ranges from $-\infty$ (IR) and $+\infty$ (UV). With this truncation the Lagrangian reduces to

$$
\begin{aligned}
L=\sqrt{-g}\{ & -\frac{1}{4} R+\frac{1}{2}(\partial m)^{2}+\frac{1}{2}(\partial \sigma)^{2} \\
& \left.-\frac{3}{8}\left[\left(\cosh \frac{2 m}{\sqrt{3}}\right)^{2}+4 \cosh \frac{2 m}{\sqrt{3}} \cosh 2 \sigma-(\cosh 2 \sigma)^{2}+4\right]\right\} .
\end{aligned}
$$

Because of supersymmetry, the fields $\phi, m$ and $\sigma$ satisfy the first order equations

$$
\begin{aligned}
\dot{\phi} & =\frac{1}{2}\left[\cosh \frac{2 m}{\sqrt{3}}+\cosh 2 \sigma\right], \\
\dot{m} & =-\frac{\sqrt{3}}{2} \sinh \frac{2 m}{\sqrt{3}}, \\
\dot{\sigma} & =-\frac{3}{2} \sinh 2 \sigma,
\end{aligned}
$$

descending form the superpotential

$$
W=\frac{3}{4}\left[\cosh \frac{2 m}{\sqrt{3}}+\cosh 2 \sigma\right] .
$$

The solution, which is often denoted as GPPZ flow [6], is

$$
\begin{aligned}
m(y) & =\frac{\sqrt{3}}{2} \log \left[\frac{1+e^{-\left(y-C_{1}\right)}}{1-e^{-\left(y-C_{1}\right)}}\right]=\sqrt{3} \operatorname{arctanh} e^{-\left(y-C_{1}\right)} \\
\sigma(y) & =\frac{1}{2} \log \left[\frac{1+e^{-3\left(y-C_{2}\right)}}{1-e^{-3\left(y-C_{2}\right)}}\right]=\operatorname{arctanh} e^{-3\left(y-C_{2}\right)} \\
\phi(y) & =y+\frac{1}{2} \log \left[1-e^{-2\left(y-C_{1}\right)}\right]+\frac{1}{6} \log \left[1-e^{-6\left(y-C_{2}\right)}\right] \\
& =y-\log \cosh \frac{m(y)}{\sqrt{3}}-\frac{1}{3} \log \cosh \sigma(y) .
\end{aligned}
$$

where $C_{1}$ and $C_{2}$ are two arbitrary integration constants. ${ }^{9}$

\footnotetext{
${ }^{9}$ The integration constants $C_{i}$ used here are identical to those used by Pilch \& Warner [20], and are related to those used by GPPZ [6] by $C_{1}^{(\mathrm{GPPZ})}=C_{1}, C_{2}^{(\mathrm{GPPZ})}=3 C_{2}$. Also the definition of $\phi(y)$ differs between Pilch \& Warner and GPPZ. Here we are using the Pilch \& Warner definition, which is related to GPPZ by $\phi^{(G P P Z)}=\phi-\left(C_{1}+C_{2}\right) / 2$.
} 
Generically, solutions of the type (2.12) can represent both deformations of the dual field theory by an operator $\mathcal{O}$ and/or different vacua of the same theory characterised by a vev $\langle\mathcal{O}\rangle$. The behaviour of the solution in the asymptotic AdS region, $y \rightarrow+\infty$, discriminates between the two options. For $y \rightarrow+\infty$, the asymptotic behaviour consists of a non-normalisable part and a normalisable one

$$
\varphi \underset{y \rightarrow+\infty}{\sim} e^{(\Delta-4) y}(A+\cdots)+e^{-\Delta y}(B+\cdots),
$$

where $\Delta$ is the conformal dimension of the dual operator and the dots in the leading non-normalizable part are local functions of $A$ while the dots in the normalizable part are functions of both $A$ and $B$. The coefficient $A$ of the non-normalisable solution is interpreted as a deformation of the Lagrangian while the coefficient $B$ of the normalisable solution is related to the vev $B=1 /(2 \Delta-4)\langle O\rangle$, where $O$ is the operator dual to $\varphi$ [28].

For $y \rightarrow+\infty$, the GPPZ solution behaves as

$$
\begin{array}{ll}
\phi(y) \underset{y \rightarrow+\infty}{\sim} y & m_{0}=\sqrt{3} e^{C_{1}} \\
m(y) \underset{y \rightarrow+\infty}{\sim} m_{0} e^{-y}, & \sigma_{0}=2 e^{3 C_{2}} . \\
\sigma(y) \underset{y \rightarrow+\infty}{\sim} \frac{1}{2} \sigma_{0} e^{-3 y}, &
\end{array}
$$

From these asymptotics we see that, since $\Delta=3, m_{0}$ corresponds to a mass deformation and $\sigma_{0}=\operatorname{Re}\langle\lambda \lambda\rangle$ is the real part of the gaugino condensate. It is then natural to interpret the solution as a flow from the mass deformed $\mathcal{N}=4$ to $\mathcal{N}=1^{*}$ in the IR.

The metric has a naked singularity for $y \rightarrow C_{1}$ (with $y \geq C_{1}$ ),

$$
d s^{2}=d y^{2}+a\left(y-C_{1}\right) d x^{\mu} d x_{\mu}+\ldots,
$$

where $a=2 e^{C_{1}+C_{2}}\left(2 \sinh \left(3\left(C_{1}-C_{2}\right)\right)\right)^{1 / 3}$. The Ricci scalar is singular

$$
R=-\left(y-C_{1}\right)^{-2}+\ldots
$$

and there is no change of frame in which the singularity disappears or is milder. Notice that also the solution for $m$ diverges at $y=C_{1}$

$$
m(y)=-\frac{\sqrt{3}}{2} \log \left(y-C_{1}\right)+\ldots
$$

while the behaviour of $\sigma$ depends on the relation between $C_{1}$ and $C_{2}$. If $C_{2} \leq C_{1}$ then $\sigma$ is regular.

Singularities of this kind are common in most $5 d$ solutions and criteria have been proposed to establish whether the solutions are physically acceptable or not. In particular in [33] it was proposed that a singular solution is physically acceptable if it can be obtained as the zero temperature limit of a regular black-hole. The conditions for the existence of the black hole solution constrain the parameters of the singular solution. In this case the criterion gives $C_{2} \leq C_{1}$. By looking at the behaviour of Wilson loops it was shown in [6] that the solutions with $C_{2} \leq C_{1}$ confines. Such solutions should then be dual to the confining vacua of $\mathcal{N}=1^{*}$. 


\section{Uplift of the GPPZ solution}

The general uplift of $\mathcal{N}=8 \mathrm{SO}(6)$ gauged supergravity to type IIB was constructed in [9] and we recall the main formulae in appendix A. In this section we first review the 4-scalar truncation of $\mathcal{N}=8$ supergravity in which the GPPZ solution lives and then we apply to it the uplift formulae of appendix A to obtain the full IIB uplift of the GPPZ solution. Finally, we explicitly verify the entire set of IIB field equations is satisfied by the ten-dimensional solution.

\subsection{Four-scalar truncation of $D=5$ supergravity}

As discussed above, an important ingredient in the construction of the GPPZ solution is the invariance under an $\mathrm{SO}(3)$ subgroup of the gauge group $\mathrm{SO}(6)$ that allows to truncate the full theory to a pair of complex scalars [6]. Even if the GPPZ solution was found in a truncation involving only two real scalars, one can actually embed the flow in a larger theory that is obtained by truncating the $\mathcal{N}=8$ supergravity to the full set of $\mathrm{SO}(3)$ invariant fields [20]. This gives an $\mathcal{N}=2$ supergravity coupled to two hyper-multiplets. Of the 42 scalars $(2.1)$ of the $\mathcal{N}=8$ theory we only keep the 8 singlets under the

$$
\mathrm{SO}(3)_{\operatorname{diag}} \subset \mathrm{SO}(3) \times \mathrm{SO}(3) \subset \mathrm{SO}(6)
$$

subgroup of the gauge group $\mathrm{SO}(6) \sim \mathrm{SU}(4)$. These form the coset space $G_{2(2)} / \mathrm{SO}(4)$ and are dual to the operators

$$
\begin{aligned}
& \mathcal{O}_{1}=\sum_{i=1}^{3}\left(\operatorname{tr}\left(\phi_{i} \phi_{i}\right)-\operatorname{tr}\left(\phi_{i+3} \phi_{i+3}\right)\right), \\
& \mathcal{O}_{2}=\sum_{i=1}^{3} \operatorname{tr}\left(\phi_{i} \phi_{i+3}\right) \\
& \mathcal{O}_{3}=\sum_{i=1}^{3} \operatorname{tr}\left(\lambda_{i} \lambda_{i}\right) \\
& \mathcal{O}_{4}=\operatorname{tr}\left(\lambda_{4} \lambda_{4}\right) \\
& \mathcal{O}_{5}=\left(F^{+}\right)^{2} \text {, } \\
& \mathcal{O}_{6}=\left(F^{-}\right)^{2},
\end{aligned}
$$

where $F^{ \pm}$is the (anti)-self-dual field gauge strength. $\mathcal{O}_{1}$ and $\mathcal{O}_{2}$ are the $\mathrm{SO}(3)_{\text {diag }}$ singlets contained in $\mathbf{2 0}^{\prime}$, the complex operators $\mathcal{O}_{3}$ and $\mathcal{O}_{4}$ are the $\mathrm{SO}(3)_{\text {diag }}$ singlets in the $\mathbf{1 0}$ and $\overline{\mathbf{1 0}}$, and $\mathcal{O}_{5}, \mathcal{O}_{6}$ correspond to the two singlets. Among the $\mathrm{SU}(4)_{R}$ gauge fields, the truncation to singlets under (3.1) only keeps a single $\mathrm{U}(1)$ gauge field, dual to the $\mathrm{U}(1)_{R}$ subgroup of $\mathrm{SU}(4)_{R} \rightarrow \mathrm{SU}(3) \times \mathrm{U}(1)_{R} \cdot{ }^{10}$

The further truncation to the 2 complex scalars dual to $\mathcal{O}_{3}$ and $\mathcal{O}_{4}$ can also be shown to be consistent as it corresponds to the truncation to singlets under an additional discrete subgroup within $\mathrm{U}(1)_{R} \times \mathrm{U}(1)_{Y}$, see [20] for details. In field theory the discrete $\mathrm{U}(1)_{R}$ transformation is

$$
\left(\phi_{i}, \phi_{i+3}\right) \rightarrow\left(\phi_{i+3},-\phi_{i}\right), \quad \lambda_{i} \rightarrow e^{-\frac{\pi}{4} i} \lambda_{i}, \quad \lambda_{4} \rightarrow e^{\frac{3 \pi}{4} i} \lambda_{4}
$$

\footnotetext{
${ }^{10}$ We normalize the $\mathrm{U}(1)$ such that the charges are those of the QFT, see the discussion around (6.88) in [15].
} 
Thus under this transformation

$$
\mathcal{O}_{1} \rightarrow-\mathcal{O}_{1}, \quad \mathcal{O}_{2} \rightarrow-\mathcal{O}_{2}, \quad \mathcal{O}_{3} \rightarrow e^{-\frac{\pi}{2} i} \mathcal{O}_{3}, \quad \mathcal{O}_{4} \rightarrow e^{-\frac{\pi}{2} i} \mathcal{O}_{4}
$$

while $\mathcal{O}_{5}$ and $\mathcal{O}_{6}$ are invariant. Under $\mathrm{U}(1)_{Y}$ the operators $\mathcal{O}_{1}$ and $\mathcal{O}_{2}$ are neutral, $\mathcal{O}_{3}$ and $\mathcal{O}_{4}$ have charge 2 (and the complex conjugates charge -2 ) and $\mathcal{O}_{5}$ and $\mathcal{O}_{6}$ have charges 4 and -4 (see (2.1)). Thus the combined operation of the discrete $\mathrm{U}(1)_{R}$ in (3.4) and an discrete $\mathrm{U}(1)_{Y}$ rotation with angle $\pi / 4$ yields

$$
\mathcal{O}_{1} \rightarrow-\mathcal{O}_{1}, \quad \mathcal{O}_{2} \rightarrow-\mathcal{O}_{2}, \quad \mathcal{O}_{3} \rightarrow \mathcal{O}_{3}, \quad \mathcal{O}_{4} \rightarrow \mathcal{O}_{4}, \quad \mathcal{O}_{5} \rightarrow-\mathcal{O}_{5}, \quad \mathcal{O}_{6} \rightarrow-\mathcal{O}_{6}
$$

thus projecting out $\mathcal{O}_{1}, \mathcal{O}_{2}, \mathcal{O}_{5}, \mathcal{O}_{6}$ and keeping $\mathcal{O}_{3}$ and $\mathcal{O}_{4}$.

We parameterise the scalars dual to $\mathcal{O}_{3}$ and $\mathcal{O}_{4}$ as

$$
\underline{m}=m e^{i \varphi}, \quad \underline{\sigma}=\sigma e^{i \omega} .
$$

The five-dimensional theory [34] then reduces to

$$
\begin{aligned}
\frac{1}{\sqrt{g}} \mathcal{L}= & -\frac{1}{4} R-\frac{1}{12} F_{\mu \nu} F^{\mu \nu}-\frac{1}{54} \epsilon^{\mu \nu \rho \sigma \tau} A_{\mu} F_{\nu \rho} F_{\sigma \tau}+\frac{1}{2} \partial_{\mu} m \partial^{\mu} m+\frac{1}{2} \partial_{\mu} \sigma \partial^{\mu} \sigma \\
& +\frac{3}{8} \sinh ^{2}\left(\frac{2 m}{\sqrt{3}}\right) D_{\mu} \varphi D^{\mu} \varphi+\frac{1}{8} \sinh ^{2}(2 \sigma) D_{\mu} \omega D^{\mu} \omega-V_{\text {pot }}
\end{aligned}
$$

with the Maxwell and Chern-Simons terms of minimal supergravity, covariant derivatives

$$
D_{\mu} \omega=\partial_{\mu} \omega+2 A_{\mu}, \quad D_{\mu} \varphi=\partial_{\mu} \varphi+\frac{2}{3} A_{\mu},
$$

and the scalar potential

$$
V_{\text {pot }}=-\frac{3}{8}\left(4 \cosh \left(\frac{2 m}{\sqrt{3}}\right) \cosh (2 \sigma)+\cosh ^{2}\left(\frac{2 m}{\sqrt{3}}\right)-\cosh ^{2}(2 \sigma)+4\right),
$$

which only depends on the absolute values of the complex scalars. The scalar kinetic term is an $(\mathrm{SU}(1,1) / \mathrm{U}(1))^{2}$ coset space, and the covariant derivatives (3.8) correspond to the gauging of $\mathrm{U}(1)_{R}$

Note that the angles $\varphi, \omega$ source the Maxwell equation

$$
\begin{aligned}
\nabla_{\nu} F^{\mu \nu}+\frac{1}{6} \epsilon^{\mu \nu \rho \sigma \tau} F_{\nu \rho} F_{\sigma \tau} & =\frac{3}{2} \sinh ^{2}\left(\frac{2 m}{\sqrt{3}}\right) D_{\mu} \varphi+\frac{3}{2} \sinh ^{2}(2 \sigma) D_{\mu} \omega \\
& \equiv \frac{3}{2} \mathcal{J}^{\mu} .
\end{aligned}
$$

Thus one may either set the vector field to zero and consider constant angles or demand that the angles are covariantly constant,

$$
D_{\mu} \varphi=D_{\mu} \omega=0
$$

This condition is solved by

$$
\omega=-\lambda, \quad \varphi=-\frac{1}{3} \lambda, \quad A_{\mu}=\frac{1}{2} \partial_{\mu} \lambda,
$$

for any spacetime dependent function $\lambda(x)$. 
With the angles being (covariantly) constant, the field equations for $m$ and $\sigma$ decouple and the GPPZ flow (2.12) is still a solution. In the uplift formulae we will also employ the variables [20]

$$
\mu \equiv e^{\sigma}, \quad \nu=e^{m / \sqrt{3}},
$$

in terms of which the flow equations (2.10) take the form

$$
\begin{aligned}
\dot{\mu} & =\frac{3}{4 \mu}\left(1-\mu^{4}\right), \\
\dot{\nu} & =\frac{1}{4 \nu}\left(1-\nu^{4}\right), \\
\dot{\phi} & =\frac{1}{4 \mu^{2}}\left(1+\mu^{4}\right)+\frac{1}{4 \nu^{2}}\left(1+\nu^{4}\right) .
\end{aligned}
$$

\subsection{Uplift of the 4-scalar truncation: metric and dilaton/axion}

In order to apply the explicit uplift formulae given in appendix A, we first evaluate the matrix (A.1) for the four-scalar truncation $(\mu, \nu, \varphi, \omega)$ by exponentiating the associated generators in the group $E_{6(6)}$. We give some details in appendix B. Since all scalars are singlets of the $\mathrm{SO}(3)$ in (3.1) it proves useful to decompose the $S^{5}$ sphere harmonics $\mathcal{Y}^{a}$ into

$$
\mathcal{Y}^{a} \longrightarrow\left\{u^{i}, v^{i}\right\}
$$

with $u^{i} u^{i}+v^{i} v^{i}=1$. Moreover, for compactness of notation, it is useful to define the rotated functions

$$
\begin{aligned}
U^{i} & =\cos \left(\frac{1}{4}(\varphi+\omega)\right) u^{i}+\sin \left(\frac{1}{4}(\varphi+\omega)\right) v^{i}, \\
V^{i} & =\cos \left(\frac{1}{4}(\varphi+\omega)\right) v^{i}-\sin \left(\frac{1}{4}(\varphi+\omega)\right) u^{i},
\end{aligned}
$$

where $\varphi$ and $\omega$ are the $x$-dependent phases of the scalars $m$ and $\sigma$ of the $D=5$ theory, (3.6). This transformation is a local $\mathrm{U}(1)$ corresponding to the $\mathrm{U}(1)_{R}$ of the dual QFT. Similarly, we define the rotated one-forms

$$
\begin{aligned}
\Theta^{i} & =\cos \left(\frac{1}{4}(\varphi+\omega)\right) D u^{i}+\sin \left(\frac{1}{4}(\varphi+\omega)\right) D v^{i}, \\
\Lambda^{i} & =\cos \left(\frac{1}{4}(\varphi+\omega)\right) D v^{i}-\sin \left(\frac{1}{4}(\varphi+\omega)\right) D u^{i},
\end{aligned}
$$

where the covariant derivatives

$$
\begin{aligned}
D u^{i} & \equiv d u^{i}-\frac{1}{3} v^{i} A_{\mu} d x^{\mu} \\
D v^{i} & \equiv d v^{i}+\frac{1}{3} u^{i} A_{\mu} d x^{\mu}
\end{aligned}
$$

correspond to the Kaluza-Klein basis (A.13). These are the objects that naturally appear in the uplift formulae of appendix A. Let us also note that the proper identification of the 
$\mathrm{U}(1)$ vector field $A_{\mu}$ among the $15 \mathrm{SO}(6)$ fields $A_{\mu}^{a b}$ gives rise to the relations

$$
\begin{aligned}
F_{\mu \nu}^{a b} M_{a b, c d} F^{\mu \nu c d} & =\frac{4}{3} F_{\mu \nu} F^{\mu \nu}, \\
\varepsilon_{a b c d e f} F_{\mu \nu}^{a b} F_{\rho \sigma}^{c d} A_{\tau}^{e f} & =-\frac{32 \sqrt{2}}{9} F_{\mu \nu} F_{\rho \sigma} A_{\tau} .
\end{aligned}
$$

We can now give the fields of the uplifted solution. The IIB metric (A.7) takes the explicit form

$$
d s_{\mathrm{IIB}}^{2}=\Delta^{-2 / 3}\left(g_{\mu \nu}(x) d x^{\mu} d x^{\nu}+\Delta^{8 / 3} d \hat{s}_{5}^{2}\right)
$$

with the warp factor $\Delta$ and the internal metric $d \hat{s}_{5}^{2}$ given by

$$
\begin{aligned}
\Delta^{-8 / 3}= & \frac{\left(1+\mu^{2} \nu^{2}\right)^{3}\left(\mu^{2}+\nu^{6}\right)}{16 \mu^{4} \nu^{6}}+\frac{U^{2} V^{2}}{16 \mu^{4} \nu^{8}}\left(1-\nu^{4}\right)^{2}\left(\mu^{2}-\nu^{2}\right)^{2}\left(1+\mu^{2} \nu^{2}\right)^{2} \\
& -\frac{(U \cdot V)^{2}}{16 \mu^{4} \nu^{8}}\left(1-\nu^{4}\right)^{2}\left(1-\mu^{2} \nu^{2}\right)^{2}\left(\mu^{2}+\nu^{2}\right)^{2}, \\
d \hat{s}_{5}^{2}= & \frac{\left(1+\nu^{4}\right)\left(\mu^{2}+\nu^{2}\right)\left(1+\mu^{2} \nu^{2}\right)}{8 \mu^{2} \nu^{4}}\left(\Theta^{i} \Theta^{i}+\Lambda^{i} \Lambda^{i}\right) \\
& -\frac{\left(1-\nu^{4}\right)^{2}}{8 \nu^{4}}\left(\left(U^{2}-V^{2}\right)\left(\Theta^{i} \Theta^{i}-\Lambda^{i} \Lambda^{i}\right)+4(U \cdot V) \Theta^{i} \Lambda^{i}\right) \\
& +\frac{\left(1-\mu^{4}\right)\left(1-\nu^{4}\right)}{8 \mu^{2} \nu^{2}}\left(\left(U^{2}-V^{2}\right)\left(\Theta^{i} \Theta^{i}-\Lambda^{i} \Lambda^{i}\right)-4(U \cdot V) \Theta^{i} \Lambda^{i}\right) \\
& +\frac{\left(1-\mu^{4} \nu^{4}\right)\left(1-\mu^{2} \nu^{2}\right)\left(\mu^{2}+\nu^{6}\right)}{16 \mu^{4} \nu^{6}}\left(\left(V^{i} \Theta^{i}\right)\left(V^{j} \Theta^{j}\right)+\left(U^{i} \Lambda^{i}\right)\left(U^{j} \Lambda^{j}\right)\right) \\
& +\frac{\left(1-\mu^{4} \nu^{4}\right)\left(1+\mu^{2} \nu^{2}\right)\left(\mu^{2}-\nu^{6}\right)}{8 \mu^{4} \nu^{6}}\left(V^{i} \Theta^{i}\right)\left(U^{j} \Lambda^{j}\right) \\
& -\frac{\left(\mu^{4}-\nu^{4}\right)\left(1-\nu^{8}\right)}{4 \mu^{2} \nu^{6}}\left(U^{i} \Theta^{i}\right)\left(V^{j} \Lambda^{j}\right) .
\end{aligned}
$$

For vanishing angles (i.e. $U^{i}=u^{i}, V^{i}=v^{i}, \Theta^{i}=d u^{i}, \Lambda^{i}=d v^{i}$ ) we recover the result from [20]. ${ }^{11}$ It is important to note that the only singularities of the IIB metric can be located at $\mu, \nu=0$ or $\mu, \nu=\infty$. Indeed, the warp factor (3.21) can be estimated (using that $U^{2} V^{2} \geq(U \cdot V)^{2}$, and $\left.U^{2} V^{2}=U^{2}\left(1-U^{2}\right) \leq \frac{1}{4}\right)$ to be

$$
\begin{aligned}
\Delta^{-8 / 3} & \geq \frac{\left(1+\mu^{2} \nu^{2}\right)^{3}\left(\mu^{2}+\nu^{6}\right)}{16 \mu^{4} \nu^{6}}-\frac{U^{2} V^{2}}{4 \mu^{2} \nu^{6}}\left(1-\mu^{4}\right)\left(1-\nu^{4}\right)^{3} \\
& \geq \frac{\left(1+\mu^{2} \nu^{2}\right)^{3}\left(\mu^{2}+\nu^{6}\right)}{16 \mu^{4} \nu^{6}}-\frac{1}{16 \mu^{2} \nu^{6}}\left(1-\mu^{4}\right)\left(1-\nu^{4}\right)^{3} \\
& =\frac{\left(\mu^{2}+\nu^{2}\right)^{3}\left(1+\mu^{2} \nu^{6}\right)}{16 \mu^{4} \nu^{6}}>0 .
\end{aligned}
$$

We will take a closer look at the possible singularities in section 5 .

\footnotetext{
${ }^{11}$ We corrected a typo in [20] in the form of index contractions of the penultimate term in (3.22).
} 
For the symmetric $\mathrm{SL}(2)$ dilaton/axion matrix $m_{\alpha \beta}$,

$$
m_{\alpha \beta}=\frac{1}{\Im \tau}\left(\begin{array}{cc}
|\tau|^{2} & -\Re \tau \\
-\Re \tau & 1
\end{array}\right), \quad \tau=C_{0}+i e^{-\Phi}
$$

the uplift formula (A.10) yields

$$
m_{\alpha \beta}=\Delta^{4 / 3} \mathcal{S}_{\alpha}{ }^{a} \mathcal{S}_{\beta}{ }^{b} \mathrm{~m}_{a b},
$$

where $S$ is an $\mathrm{SO}(2)$ rotation matrix parameterised by

$$
\mathcal{S}=\left(\begin{array}{cc}
\cos \left(\frac{3}{4} \varphi-\frac{1}{4} \omega\right) & \sin \left(\frac{3}{4} \varphi-\frac{1}{4} \omega\right) \\
-\sin \left(\frac{3}{4} \varphi-\frac{1}{4} \omega\right) & \cos \left(\frac{3}{4} \varphi-\frac{1}{4} \omega\right)
\end{array}\right)
$$

and $\mathrm{m}_{a b}$ is a $G L(2)$ matrix with entries

$$
\begin{aligned}
& \mathrm{m}_{11}=\frac{1+\mu^{2} \nu^{2}}{8 \mu^{2} \nu^{4}}\left(\left(1+\nu^{4}\right)\left(\mu^{2}+\nu^{2}\right)+\left(1-\nu^{4}\right)\left(\mu^{2}-\nu^{2}\right)\left(U^{2}-V^{2}\right)\right), \\
& \mathrm{m}_{12}=\frac{\left(1-\mu^{2} \nu^{2}\right)\left(1-\nu^{4}\right)\left(\mu^{2}+\nu^{2}\right)}{4 \mu^{2} \nu^{4}}(U \cdot V), \\
& \mathrm{m}_{22}=\frac{1+\mu^{2} \nu^{2}}{8 \mu^{2} \nu^{4}}\left(\left(1+\nu^{4}\right)\left(\mu^{2}+\nu^{2}\right)-\left(1-\nu^{4}\right)\left(\mu^{2}-\nu^{2}\right)\left(U^{2}-V^{2}\right)\right) .
\end{aligned}
$$

It is straightforward to check that the determinant of $\mathrm{m}_{a b}$ is given by $\Delta^{-8 / 3}(3.21)$ as required in order to have $m_{\alpha \beta} \in \mathrm{SL}(2)$. Again, for vanishing angles we recover the result from $[20]$.

It is remarkable that the dependence of the IIB axion/dilation on the (a priori $x$ dependent) 5D angles $(\varphi, \omega)$ is entirely captured by a rotation of the internal coordinates (3.16) and the $\mathrm{SO}(2) \subset \mathrm{SL}(2)_{\mathrm{IIB}}$ rotation (3.26). ${ }^{12}$ We will see in the following that this feature persists for the full IIB uplift. In particular, $2 \pi$ periodicity of the $5 D$ theory implies that the IIB uplift is invariant under the combination of an exchange $U^{i} \leftrightarrow V^{i}$ with a constant $\mathrm{SL}(2)$ rotation (3.26) with $\mathcal{S}=-i \sigma_{2}$, as is easily verified for (3.22) and (3.27).

\subsection{Uplift of the 4-scalar truncation: $p$-forms}

We now evaluate (A.11) on the four scalar truncation in order to derive the IIB $p$-forms. For the two-form doublet, we find

$$
C_{m n \alpha}=\Delta^{8 / 3} \mathcal{S}_{\alpha}{ }^{a} \mathrm{C}_{m n a},
$$

\footnotetext{
${ }^{12}$ Note that when evaluated on (3.12) the $\mathrm{SO}(2) \subset \mathrm{SL}(2)_{\text {IIB }}$ becomes the identity and $\Theta^{i}=d U^{i}, \Lambda^{i}=d V^{i}$ (see (3.17)) and thus the 10 dimensional solution involving a non-trivial $\lambda(x)$ can be obtained by simply substituting $u^{i}, v^{i}$ with $U^{i}, V^{i}$.
} 
where $\mathcal{S}$ is the $\mathrm{SO}(2)$ rotation matrix in (3.26), and the 2-form $\mathrm{C}_{a}$ are

$$
\begin{aligned}
\mathrm{C}_{1}= & b_{1} \varepsilon^{i j k}( \\
& \left(1-\mu^{2} \nu^{2}\right)\left(\mu^{2}+\nu^{6}\right) V^{i} \Theta^{j} \wedge \Theta^{k}+\left(1+\mu^{2} \nu^{2}\right)\left(\mu^{2}-\nu^{6}\right) V^{i} \Lambda^{j} \wedge \Lambda^{k} \\
& \left.+2 \nu^{2}\left(1-\mu^{4} \nu^{4}\right) U^{i} \Theta^{j} \wedge \Lambda^{k}\right) \\
& +b_{2} \varepsilon^{i j k}\left(\left(1+\mu^{2} \nu^{2}\right)\left(\mu^{2}-\nu^{6}\right) U^{i} \Theta^{j} \wedge \Theta^{k}+\left(1-\mu^{2} \nu^{2}\right)\left(\mu^{2}+\nu^{6}\right) U^{i} \Lambda^{j} \wedge \Lambda^{k}\right. \\
& \left.+2 \nu^{2}\left(1-\mu^{4} \nu^{4}\right) V^{i} \Theta^{j} \wedge \Lambda^{k}\right) \\
& \\
\mathrm{C}_{2}=- & \left.\mathrm{C}_{1}\right|_{U^{i} \leftrightarrow V^{i}, \Theta^{i} \leftrightarrow \Lambda^{i}},
\end{aligned}
$$

with the functions

$$
\begin{aligned}
& b_{1}=-\frac{1+\mu^{2} \nu^{2}}{64 \mu^{4} \nu^{8}}\left(\left(1+\nu^{4}\right)\left(\mu^{2}+\nu^{2}\right)+\left(1-\nu^{4}\right)\left(\mu^{2}-\nu^{2}\right)\left(U^{2}-V^{2}\right)\right), \\
& b_{2}=\frac{1-\mu^{2} \nu^{2}}{32 \mu^{4} \nu^{8}}\left(\left(1-\nu^{4}\right)\left(\mu^{2}+\nu^{2}\right)(U \cdot V)\right) .
\end{aligned}
$$

Again, the dependence on the $5 D$ angles $(\varphi, \omega)$ is entirely captured by the $\mathrm{SO}(2)$ rotation (3.26) and the rotated basis (3.16). The internal component of the 4 -form potential takes the form

$$
\begin{aligned}
C= & \stackrel{\circ}{C}+\frac{1}{4 !} \Delta^{8 / 3} \varepsilon_{i j m} \varepsilon_{k l n}\left(f_{1}\left(U^{m} U^{n}-V^{m} V^{n}\right)+2 f_{2} U^{(m} V^{n)}\right) \Theta^{i} \wedge \Theta^{j} \wedge \Lambda^{k} \wedge \Lambda^{l} \\
& +\frac{1}{4 !} \Delta^{8 / 3} f_{3} \Theta^{i} \wedge \Theta^{j} \wedge \Lambda^{i} \wedge \Lambda^{j}
\end{aligned}
$$

where the background field $\stackrel{\circ}{C}$ is given in (A.5) and

$$
\begin{aligned}
& f_{1}=-\frac{3(U \cdot V)}{32 \mu^{4} \nu^{8}}\left(1-\nu^{4}\right)^{2}\left(\mu^{2}+\nu^{2}\right)^{2}\left(1-\mu^{2} \nu^{2}\right)^{2}, \\
& f_{2}=\frac{3\left(U^{2}-V^{2}\right)}{64 \mu^{4} \nu^{8}}\left(1-\nu^{4}\right)^{2}\left(\mu^{2}-\nu^{2}\right)^{2}\left(1+\mu^{2} \nu^{2}\right)^{2}, \\
& f_{3}=\frac{3(U \cdot V)\left(U^{2}-V^{2}\right)}{8 \mu^{2} \nu^{6}}\left(1-\nu^{4}\right)^{3}\left(1-\mu^{4}\right) .
\end{aligned}
$$

Finally, the external component $C_{\mu \nu \rho \sigma}$ is determined from integrating the $y$-independent function $^{13}$

$$
5 \partial_{[\mu} C_{\nu \rho \sigma \tau]}=-\frac{1}{3} \omega_{\mu \nu \rho \sigma \tau}\left(V_{\mathrm{pot}}-\frac{1}{6} F_{\kappa \lambda} F^{\kappa \lambda}\right)-\frac{20 \sqrt{2}}{9} F_{[\mu \nu} F_{\rho \sigma} A_{\tau]},
$$

with the scalar potential $V_{\text {pot }}$ from (3.9).

\subsection{Five-form field strength and self-duality equations}

As a first consistency check, we compute the IIB 5-form field strength

$$
F_{\hat{\mu}_{1} \ldots \hat{\mu}_{5}} \equiv 5 \partial_{\left[\hat{\mu}_{1}\right.} C_{\left.\hat{\mu}_{2} \ldots \hat{\mu}_{5}\right]}-\frac{15}{4} \varepsilon_{\alpha \beta} C_{\left[\hat{\mu}_{1} \hat{\mu}_{2}\right.}{ }^{\alpha} \partial_{\hat{\mu}_{3}} C_{\left.\hat{\mu}_{4} \hat{\mu}_{5}\right]}^{\beta},
$$

\footnotetext{
${ }^{13}$ Here, and in the following, we use the notation $\omega_{\mu \nu \rho \sigma \tau}=\sqrt{|g|} \varepsilon_{\mu \nu \rho \sigma \tau}$ for the 5D volume form.
} 
and verify that it satisfies the first order self-duality equations

$$
F=\star F .
$$

Here and in the following, indices $\hat{\mu}$ refer to the ten-dimensional coordinates, split as $\left\{x^{\hat{\mu}}\right\}=\left\{x^{\mu}, y^{m}\right\}$. After some computation we find that the internal components $F_{m_{1} \ldots m_{5}}$ calculated from the above expressions for $C_{m n}{ }^{\alpha}$ and $C_{k l m n}$, take the compact form

$$
F_{m_{1} \ldots m_{5}}=-\frac{1}{3} \Delta^{8 / 3} \stackrel{\circ}{\omega}_{m_{1} \ldots m_{5}} V_{\text {pot }},
$$

with $V_{\text {pot }}$ the scalar potential in (3.9), and ${\stackrel{\circ}{m_{1} \ldots m_{5}}}_{5}$ is the volume-form of $S^{5}$ defined in equation (A.5). The external component of the five-form field strength $F_{\mu \nu \rho \sigma \tau}$ is computed from (3.33), (3.34) as

$$
\begin{aligned}
F_{\mu \nu \rho \sigma \tau}= & 5 \partial_{[\mu} C_{\nu \rho \sigma \tau]}-10 F_{[\mu \nu}{ }^{m} C_{\rho \sigma \tau] m} \\
= & -\frac{1}{3} \omega_{\mu \nu \rho \sigma \tau}\left(V_{\text {pot }}-\frac{1}{12} F_{\kappa \lambda} F^{\kappa \lambda}\right)-\frac{2}{9} F_{[\mu \nu} F_{\rho \sigma} A_{\tau]} \\
& +\frac{5}{16} F_{[\mu \nu}{ }^{g a} \mathcal{Y}^{g} \mathcal{Y}^{b}\left(4 \omega_{\rho \sigma \tau] \kappa \lambda} M_{a b, c d} F^{\kappa \lambda c d}+3 \sqrt{2} \varepsilon_{a b c d e f} F_{\rho \sigma}{ }^{c d} A_{\tau]}{ }^{e f}\right) \\
= & -\frac{1}{3} \omega_{\mu \nu \rho \sigma \tau} V_{\text {pot }},
\end{aligned}
$$

upon using (3.19). Comparing to (3.36), we find that five-form we have is indeed self-dual.

Similar calculations lead to the other components of the five-form

$$
F_{\mu m_{1} \ldots m_{4}} D y^{m_{1}} \wedge D y^{m_{2}} \wedge D y^{m_{3}} \wedge D y^{m_{4}}=3 \Delta \star_{5}\left(U^{i} \Lambda^{i}-V^{i} \Theta^{i}\right) \mathcal{J}_{\mu}
$$

where the current $\mathcal{J}_{\mu}$ is defined in (3.10) and the 5D Hodge dual $\star_{5}$ gives explicitly

$$
\begin{aligned}
2 \Delta^{-5 / 3} \star_{5}\left(U^{i} \Lambda^{i}-V^{i} \Theta^{i}\right)= & \Theta^{i} \wedge \Theta^{j} \wedge \Lambda^{i} \wedge \Lambda^{j} \\
& +\frac{\left(1-\nu^{4}\right)^{2}}{\nu^{4}}\left(U^{i} U^{j}+V^{i} V^{j}\right) \Theta^{i} \wedge \Theta^{k} \wedge \Lambda^{j} \wedge \Lambda^{k} .
\end{aligned}
$$

Comparing to the result for

$$
F_{\mu \nu \rho \sigma m} D y^{m}=\frac{1}{12} \omega_{\mu \nu \rho \sigma \lambda}\left(D_{\kappa} F^{\lambda \kappa}+\frac{1}{6} \omega^{\lambda \nu_{1} \ldots \nu_{4}} F_{\nu_{1} \nu_{2}} F_{\nu_{3} \nu_{4}}\right)\left(U^{i} \Lambda^{i}-V^{i} \Theta^{i}\right)
$$

shows explicitly that the IIB self-duality equations (3.35) reduce to the Maxwell equations of the $D=5$ theory (3.10). For the remaining components, we obtain after some calculation

$$
\begin{aligned}
& F_{\mu \nu \rho m n}=-\frac{1}{12} \omega_{\mu \nu \rho \sigma \tau} F^{\sigma \tau}\left(\Theta^{i} \wedge \Lambda^{i}\right)_{m n}, \\
& F_{\mu \nu k m n}=-\frac{\Delta^{1 / 3}}{12} F_{\mu \nu} \star_{5}\left(\Theta^{i} \wedge \Lambda^{i}\right)_{k m n},
\end{aligned}
$$

again in accordance with self-duality of the IIB field strength. 


\subsection{Dual 6-forms}

For an explicit check of the remaining field equations, we further truncate down to two real scalar fields, i.e. we assume constant angles and set the vector field to zero, so that in particular the IIB metric is block diagonal. This is precisely compatible with the GPPZ solution (2.12). The ten-dimensional IIB 3-form field equations take the form

$$
\nabla_{\hat{\rho}}\left(m^{\alpha \beta} F^{\hat{\mu} \hat{\nu} \hat{\rho}} \beta\right)=-\frac{2}{3} \varepsilon^{\alpha \beta} F^{\hat{\mu} \hat{\nu} \hat{\kappa} \hat{\lambda} \hat{\rho}} F_{\hat{\kappa} \hat{\lambda} \hat{\rho} \beta}
$$

and we have explicitly checked that they are verified if the five-dimensional scalar fields satisfy the five-dimensional equations of motion induced by (3.7). Rather than going through the details of this calculation, let us give an equivalent consistency check by extracting the dual six forms in ten dimensions. The field equations (3.42) may be rewritten as the Bianchi identities

$$
\partial_{\left[\hat{\rho}_{1}\right.} F_{\left.\hat{\rho}_{2} \ldots \hat{\rho}_{8}\right]}^{\alpha}=28 \varepsilon^{\alpha \beta} F_{\left[\hat{\rho}_{1} \ldots \hat{\rho}_{5}\right.} F_{\left.\hat{\rho}_{6} \hat{\rho}_{7} \hat{\rho}_{8}\right] \beta}
$$

for the dual 7 -form field strength $F_{\hat{\rho}_{1} \ldots \hat{\rho}_{7}}{ }^{\alpha}$ defined by

$$
F_{\hat{\rho}_{1} \ldots \hat{\rho}_{7}}{ }^{\alpha} \equiv \frac{1}{6} \sqrt{|G|} \varepsilon_{\hat{\rho}_{1} \ldots \hat{\rho} \hat{\rho} \hat{\mu} \hat{\nu} \hat{\rho}} m^{\alpha \beta} F^{\hat{\mu} \hat{\nu} \hat{\rho}_{\beta}}
$$

The Bianchi identities (3.43) may then be integrated to

$$
\begin{aligned}
F_{\hat{\rho}_{1} \ldots \hat{\rho}_{7}}{ }^{\alpha}= & 7 \partial_{\left[\hat{\rho}_{1}\right.} C_{\left.\hat{\rho}_{2} \ldots \hat{\rho}_{7}\right]}^{\alpha}-84 \varepsilon^{\alpha \beta} C_{\left[\hat{\rho}_{1} \hat{\rho}_{2} \beta\right.} F_{\left.\hat{\rho}_{3} \ldots \hat{\rho}_{7}\right]} \\
& -70 \varepsilon^{\alpha \beta} \varepsilon^{\gamma \delta} C_{\left[\hat{\rho}_{1} \hat{\rho}_{2} \beta\right.} C_{\hat{\rho}_{3} \hat{\rho}_{4} \gamma} F_{\left.\hat{\rho}_{5} \hat{\rho}_{6} \hat{\rho}_{7}\right] \delta}
\end{aligned}
$$

in terms of the dual six-form gauge potential $C_{\hat{\rho}_{1} \ldots \hat{\rho}_{6}}{ }^{\alpha}$. With the above explicit expressions for the IIB gauge potentials (3.29) and field strengths (3.36), (3.37) of our ten-dimensional solution, we find that equations (3.44) can explicitly be integrated to give the following non-vanishing components of the six form

$$
\begin{aligned}
C_{\mu \nu \rho \sigma \tau m}{ }^{\alpha} & =\omega_{\mu \nu \rho \sigma \tau} \Xi_{m}{ }^{\alpha} \\
C_{\mu \nu \rho \sigma, m n}{ }^{\alpha} & =\omega_{\mu \nu \rho \sigma \tau} g^{\tau \lambda} \Xi_{\lambda m n}{ }^{\alpha},
\end{aligned}
$$

in terms of the one- and two-forms

$$
\begin{aligned}
\Xi^{1} & =-\frac{\left(1-\mu^{2} \nu^{2}\right)\left(\left(\mu^{4}-\nu^{4}\right)\left(1-\mu^{2} \nu^{2}\right)+2 \mu^{2} \nu^{2}\left(1+\mu^{2} \nu^{2}\right)\right)}{8 \mu^{4} \nu^{4}} \varepsilon_{i j k} U^{i} V^{j} \Lambda^{k}, \\
\Xi^{2} & =\left.\Xi^{1}\right|_{U \leftrightarrow V, \Theta \leftrightarrow \Lambda}, \\
\Xi_{\lambda}{ }^{1} & =\varepsilon_{i j k}\left(\frac{\partial_{\lambda} \nu}{\nu}-\frac{\partial_{\lambda} \mu}{3 \mu}\right) U^{i} \Theta^{j} \wedge \Theta^{k}+\varepsilon_{i j k}\left(\frac{\partial_{\lambda} \mu}{\mu}+\frac{\partial_{\lambda} \nu}{\nu}\right) U^{i} \Lambda^{j} \wedge \Lambda^{k}, \\
\Xi_{\lambda}{ }^{2} & =\left.\Xi_{\lambda}{ }^{1}\right|_{U \leftrightarrow V, \Theta \leftrightarrow \Lambda} .
\end{aligned}
$$




\subsection{Einstein equations}

It remains to check the dilaton/axion equations and the Einstein equations. In our IIB conventions, these read

$$
\nabla_{\hat{\mu}}\left(m^{\beta \gamma} \partial^{\hat{\mu}} m_{\alpha \gamma}\right)=-\frac{1}{6} m^{\beta \gamma} F_{\hat{\mu}_{1} \hat{\mu}_{2} \hat{\mu}_{3} \gamma} F_{\alpha}^{\hat{\mu}_{1} \hat{\mu}_{2} \hat{\mu}_{3}}+\frac{1}{12} \delta_{\alpha}^{\beta} m^{\gamma \delta} F_{\hat{\mu}_{1} \hat{\mu}_{2} \hat{\mu}_{3} \gamma} F^{\hat{\mu}_{1} \hat{\mu}_{2} \hat{\mu}_{3}}{ }_{\delta}
$$

and

$$
\begin{aligned}
R_{\hat{\mu} \hat{\nu}}-\frac{1}{2} G_{\hat{\mu} \hat{\nu}} R= & \frac{1}{6} F_{\hat{\mu} \hat{\kappa} \hat{\lambda} \hat{\sigma} \hat{\tau}} F_{\hat{\nu}}^{\hat{\kappa} \hat{\lambda} \hat{\sigma} \hat{\tau}}+\frac{1}{4} F_{\hat{\mu} \hat{\sigma} \hat{\tau}}{ }^{\alpha} F_{\hat{\nu}}^{\hat{\sigma} \hat{\tau} \beta} m_{\alpha \beta}-\frac{1}{24} G_{\hat{\mu} \hat{\nu}} F_{\hat{\rho} \hat{\sigma} \hat{\tau}}{ }^{\alpha} F^{\hat{\rho} \hat{\sigma} \hat{\tau} \beta} m_{\alpha \beta} \\
& -\frac{1}{4} \partial_{\hat{\mu}} m_{\alpha \beta} \partial_{\hat{\nu}} m^{\alpha \beta}+\frac{1}{8} G_{\hat{\mu} \hat{\nu}} \partial_{\hat{\rho}} m_{\alpha \beta} \partial^{\hat{\rho}} m^{\alpha \beta}
\end{aligned}
$$

It is a tedious computation to check that with the above expressions for the metric (3.20), dilaton-axion matrix (3.25), gauge potentials (3.29) and field strengths (3.36), (3.37), these field equations are indeed satisfied. We have explicitly verified all components of these equations using Mathematica [27]. We attach a Mathematica file with the explicit IIB solution and the verification of Einstein's equations in the arXiv version of this paper. Let us just note that the contribution from the five-form field strength to the energy-momentum tensor on the r.h.s. of (3.49) is simply given by

$$
\begin{aligned}
F_{\mu}{ }^{\rho_{1} \rho_{2} \rho_{3} \rho_{4}} F_{\nu \rho_{1} \rho_{2} \rho_{3} \rho_{4}} & =-\frac{8}{3} \Delta^{10 / 3} V_{\mathrm{pot}}^{2} G_{\mu \nu}, \\
F_{m}{ }^{{ }^{1} k_{2} k_{3} k_{4}} F_{n k_{1} k_{2} k_{3} k_{4}} & =\frac{8}{3} \Delta^{10 / 3} V_{\mathrm{pot}}^{2} G_{m n} .
\end{aligned}
$$

In contrast, the remaining terms on the r.h.s. of (3.49) produce very lengthy expressions in the scalars $\mu, \nu$, their derivatives, and the internal coordinates $U^{i}, V^{i}$, which we do not report in detail. They combine however precisely into the Einstein tensor computed from the metric (3.20) upon using the first order flow equations (3.14). All the ten-dimensional equations are thus satisfied.

\section{UV asymptotics of the uplifted solution}

In order to interpret our ten-dimensional solution we can compute its asymptotic behaviour for large values of the radial coordinate and check whether the various field have the falloff expected from the AdS/CFT dictionary. It is also interesting to compare our results with the asymptotic behaviours of the other supergravity solutions that are supposed to describe $\mathcal{N}=1^{*}$, namely the Polchinski-Strassler solution [5] and the zero-temperature limit of the Freedman-Minahan solution [31]. To this purpose we perform the change of variable $t=e^{C_{1}} / r$, where $r$ is the radial coordinate used in $[5,31]$. In this section we only give terms up to quadratic order in the deformation parameters $m$ and $\sigma$ and we fix the values of the angles $\varphi$ and $\omega$ to constant values. $\varphi$ and $\omega$ correspond to rotations in the space of harmonics. More general expressions can be found in appendix E. 
Dilaton/axion. The expansion takes a particularly simple form for the field $B=\frac{1+i \tau}{1-i \tau}$ that appears in the Kaluza-Klein expansion around $S^{5}$ [10]. The first terms can be easily computed for any value of the angles $\varphi$ and $\omega$ and are

$$
\begin{aligned}
B \sim & -\left(\frac{4 m_{0}^{2}}{9 r^{2}} e^{-2 i \varphi}-\frac{2 m_{0} \sigma_{0}}{3 \sqrt{3} r^{4}} e^{-i(\varphi-\omega)}\right)\left(u^{2}-v^{2}\right) \\
& -2 i\left(\frac{4 m_{0}^{2}}{9 r^{2}} e^{-2 i \varphi}+\frac{2 m_{0} \sigma_{0}}{3 \sqrt{3} r^{4}} e^{-i(\varphi-\omega)}\right)(u \cdot v) .
\end{aligned}
$$

The constants $m_{0}$ and $\sigma_{0}$ are given in (2.14) and are related to the UV mass deformation and the expectation value of gaugino condensate. The functions $\left(u^{2}-v^{2}\right)$ and $(u \cdot v)$ corresponds to $\mathrm{SO}(3)$ invariant scalar harmonics $Y^{(2,0)}$ and $Y^{(2,1)}$ on $S^{5}$ in the $\mathbf{2 0}$ of $\mathrm{SO}(6)$ (see appendix D).

From (4.1), setting the angles $\varphi$ and $\omega$ to zero, we can compute the expansions of the dilaton and axion

$$
\begin{aligned}
e^{\Phi} & \sim 1+\frac{2}{3} \frac{m_{0}^{2}}{r^{2}}\left(u^{2}-v^{2}\right)+\frac{m_{0} \sigma_{0}}{\sqrt{3} r^{4}}\left(u^{2}-v^{2}\right), \\
C_{0} & \sim \frac{4}{3} \frac{m_{0}^{2}}{r^{2}}(u \cdot v)+\frac{2}{\sqrt{3}} \frac{m_{0} \sigma_{0}}{r^{4}}(u \cdot v) .
\end{aligned}
$$

The leading behaviour of the dilaton is the same as for the zero-temperature limit of the Freedman-Minahan solution [31]. As already discussed in [31], its behaviour does not agree with the asymptotic limits of the dilaton in the Polchinski-Strassler solution [5], where the leading term of the dilaton is in a singlet of $\mathrm{SO}(6)$.

Metric. For $\varphi=\omega=0$ the large $r$ behaviour of the metric is

$$
d s_{10}^{2}=r^{2}\left(1+\frac{m_{0}^{2}}{24 r^{2}}\right) d s_{4}^{2}+\frac{d r^{2}}{r^{2}}\left(1+\frac{m_{0}^{2}}{16 r^{2}}\right)+d s_{5}^{2}
$$

where $d s_{4}^{2}$ is the flat Minkowski metric in four dimensions and the internal metric $d s_{5}^{2}$ is given by

$$
\begin{aligned}
d s_{5}^{2}= & \left(d u_{i}\right)^{2}+\left(d v_{i}\right)^{2}+d u_{i} d u_{j}\left(\frac{m_{0}^{2}}{r^{2}} A_{i j}+\frac{m_{0} \sigma_{0}}{r^{4}} B_{i j}\right) \\
& +d v_{i} d v_{j}\left(\frac{m_{0}^{2}}{r^{2}} C_{i j}+\frac{m_{0} \sigma_{0}}{r^{4}} D_{i j}\right)+d u_{i} d v_{j}\left(\frac{m_{0}^{2}}{r^{2}} E_{i j}+\frac{m_{0} \sigma_{0}}{r^{4}} F_{i j}\right)
\end{aligned}
$$

with coefficients

$$
\begin{aligned}
A_{i j} & =-\frac{1}{6}\left(3+4\left(u^{2}-v^{2}\right)\right) \delta_{i j}+\frac{1}{3} v_{i} v_{j}, \\
B_{i j} & =\frac{1}{\sqrt{3}}\left[\left(u^{2}-v^{2}\right) \delta_{i j}+v_{i} v_{j}\right], \\
C_{i j} & =-\frac{1}{6}\left(3-4\left(u^{2}-v^{2}\right)\right) \delta_{i j}+\frac{1}{3} u_{i} u_{j}, \\
D_{i j} & \left.=\frac{1}{\sqrt{3}}\left[-\left(u^{2}-v^{2}\right)\right] \delta_{i j}+u_{i} u_{j}\right],
\end{aligned}
$$




$$
\begin{aligned}
& E_{i j}=\frac{1}{3}\left[-8(u \cdot v) \delta_{i j}-8 u_{i} v_{j}+6 v_{i} u_{j}\right], \\
& F_{i j}=\frac{2}{\sqrt{3}}\left[-2(u \cdot v) \delta_{i j}+u_{i} v_{j}+v_{i} u_{j}\right] .
\end{aligned}
$$

The form of the metric agrees with the structure of the zero-temperature limit of the Freedman-Minahan solution.

Two-form potentials and field strengths. For $\varphi=\omega=0$, the first terms in the expansion of the two-forms potentials (3.28) are

$$
\begin{aligned}
C_{1}= & \frac{1}{2}\left(\frac{1}{\sqrt{3}} \frac{m_{0}}{r}+\frac{1}{2} \frac{\sigma_{0}}{r^{3}}\right) \epsilon_{i j k} v_{i} d u_{j} \wedge d u_{k}+\frac{1}{2}\left(\sqrt{3} \frac{m_{0}}{r}-\frac{1}{2} \frac{\sigma_{0}}{r^{3}}\right) \epsilon_{i j k} v_{i} d v_{j} \wedge d v_{k} \\
& +\left(\frac{1}{\sqrt{3}} \frac{m_{0}}{r}+\frac{1}{2} \frac{\sigma_{0}}{r^{3}}\right) \epsilon_{i j k} u_{i} d u_{j} \wedge d v_{k} \\
C_{2}= & -\frac{1}{2}\left(\sqrt{3} \frac{m_{0}}{r}-\frac{1}{2} \frac{\sigma_{0}}{r^{3}}\right) \epsilon_{i j k} u_{i} d u_{j} \wedge d u_{k}-\frac{1}{2}\left(\frac{1}{\sqrt{3}} \frac{m_{0}}{r}+\frac{1}{2} \frac{\sigma_{0}}{r^{3}}\right) \epsilon_{i j k} u_{i} d v_{j} \wedge d v_{k} \\
& -\left(\frac{1}{\sqrt{3}} \frac{m_{0}}{r}+\frac{1}{2} \frac{\sigma_{0}}{r^{3}}\right) \epsilon_{i j k} v_{i} d u_{j} \wedge d v_{k} .
\end{aligned}
$$

A simple derivation gives the asymptotic behaviour of the fully internal components of the field strengths $F_{i}=d C_{i}$

$$
\begin{aligned}
& F_{1}=\frac{3}{2}\left(\frac{1}{\sqrt{3}} \frac{m_{0}}{r}+\frac{1}{2} \frac{\sigma_{0}}{r^{3}}\right) \epsilon_{i j k} d u_{i} \wedge d u_{j} \wedge d v_{k}+\left(3 \sqrt{3} \frac{m_{0}}{r}-\frac{3}{2} \frac{\sigma_{0}}{r^{3}}\right) d v_{1} \wedge d v_{2} \wedge d v_{3} \\
& F_{2}=-\frac{3}{2}\left(\frac{1}{\sqrt{3}} \frac{m_{0}}{r}+\frac{1}{2} \frac{\sigma_{0}}{r^{3}}\right) \epsilon_{i j k} d u_{i} \wedge d v_{j} \wedge d v_{k}-\left(3 \sqrt{3} \frac{m_{0}}{r}-\frac{3}{2} \frac{\sigma_{0}}{r^{3}}\right) d u_{1} \wedge d u_{2} \wedge d u_{3}
\end{aligned}
$$

The terms in $1 / r$ in the (4.11) reproduce the large $r$ behaviour of the two-form potentials of the Polchinski-Strassler background [5], but the leading terms in $\sigma_{0}$ disagree. For a different values of the angles, $\varphi=\pi / 2$ and a constant arbitrary $\omega$ we also recover the leading behaviour $T=0$ limit of the three-forms in the [31].

Five-form flux. Using (3.36) it is easy to derive the large $r$ behaviour of the purely internal component of five-form flux

$$
F_{5}=-\frac{1}{5 !}\left(\frac{4}{r^{6}}-\frac{12 m_{0}^{2}}{r^{8}}\right) \epsilon_{m_{1} \ldots m_{6}} y^{m_{1}} d y^{m_{2}} \wedge d y^{m_{3}} \wedge d y^{m_{4}} \wedge d y^{m_{5}} \wedge d y^{m_{6}}
$$

where $y^{m}$ are the six coordinates of $R^{6}$ that parameterise the internal manifold. Again this expression agrees with what given by Freedman and Minahan.

\section{$5 \quad$ Singularity}

In this section we discuss the behaviour of the ten-dimensional solution as we approach the position where the five-dimensional solution has a curvature singularity.

As we reviewed in section 2 , in five dimensions we have a metric coupled to two real scalars $m$ and $\sigma$, which have a domain-wall profile along the radial direction. The 
complete solution is given in (2.12) and contains two integration constants $C_{1}$ and $C_{2}$, which parametrize the mass deformation and the gaugino condensate, respectively. The geometry is singular as the radial coordinate $y$ approaches either $C_{1}$ or $C_{2}$, as one can verify by computing curvature invariants. As in [20] we parametrize the location of the singularity by defining

$$
t=\exp \left(-\left(y-C_{1}\right)\right), \quad \chi=2 \sqrt{1-t}
$$

The singularity of the $5 d$ metric is then located at $t \rightarrow 1$ or $\chi \rightarrow 0$, and the curvature scalar and Kretschmann invariant are given by

$$
R=-\frac{16}{\chi^{4}}+O\left(\chi^{-2}\right), \quad R_{\mu \nu \rho \sigma} R^{\mu \nu \rho \sigma}=\frac{640}{\chi^{8}}+O\left(\chi^{-6}\right) .
$$

Since the scalar fields are also singular in this limit one may wonder if there is a different conformal frame than the Einstein frame, where the geometry is regular or at least less singular. It turns out that this is not the case. We will see later that the situation is different in 10 dimensions. We also define, again following [20],

$$
\lambda=e^{-3\left(C_{1}-C_{2}\right)} .
$$

It was argued in [6] that $C_{1} \geq C_{2}$ and this translates to $\lambda \leq 1$ with the equality corresponding to the case where the singularities in $m(y)$ and $\sigma(y)$ coincide. The singularity structure of the $10 d$ solution depends of whether $\lambda<1$ or $\lambda=1$ and we will discuss the two cases separately.

\subsection{The $\lambda<1$ case}

We have computed the curvature scalar of the $10 d$ solution and this has a regular limit as $\chi \rightarrow 0$,

$$
\lim _{\chi \rightarrow 0} R^{(\mathrm{full})}=\frac{\sqrt{2}}{1-\lambda^{2}} \frac{3\left(1+\lambda^{2}\right)-w_{1}^{2}\left(1-4 \lambda+\lambda^{2}\right)-w_{2}^{2}\left(1+4 \lambda+\lambda^{2}\right)}{\left(1-w_{1}^{2}-w_{2}^{2}\right)^{5 / 4}} .
$$

However, the Ricci scalar is now singular at

$$
\zeta \equiv 1-w_{1}^{2}-w_{2}^{2}=0
$$

which is precisely the ring singularity discussed in [20].

The metric near the singularity is given by

$$
\begin{array}{r}
d s^{2}=\frac{\zeta^{1 / 4}}{\sqrt{2}}\left\{\left(1+\chi^{2} f_{0}\right)\left(2 e^{2 C_{1}}\left(1-\lambda^{2}\right)^{1 / 3} \eta_{\mu \nu} d x^{\mu} d x^{\nu}+d \chi^{2}\right)+\frac{\chi^{2}}{4}\left(\frac{1}{\zeta} Y_{v}^{(0,0)^{2}}+\sigma_{2}^{2}+\sigma_{3}^{2}\right)\right. \\
\left.+\frac{1}{2 \zeta}\left(\frac{1-\lambda}{1+\lambda} d w_{1}^{2}+\frac{1+\lambda}{1-\lambda} d w_{2}^{2}\right)+\frac{\chi^{2}}{8 \zeta\left(1-\lambda^{2}\right)} \omega_{\|}+\frac{\chi^{2}}{16 \zeta^{2}\left(1-\lambda^{2}\right)^{2}} \omega_{D}\right\}+\mathcal{O}\left(\chi^{4}\right)
\end{array}
$$


where $Y_{v}^{(0,0)}$ is an $\mathrm{SO}(3)$ vector harmonic, (see appendix D). The coefficient $f_{0}$ and the differentials $\omega_{\|}$and $\omega_{D}$ are given by

$$
\begin{aligned}
f_{0}= & \lambda \frac{w_{1}^{2}-w_{2}^{2}}{4 \zeta\left(1-\lambda^{2}\right)} \\
\omega_{\|}= & \left(1+\lambda^{2}\right)(2 \zeta+1) 2 e^{2 C_{1}}\left(1-\lambda^{2}\right)^{1 / 3} \eta_{\mu \nu} d x^{\mu} d x^{\nu}+\left(2+(3 \zeta-1)\left(1-\lambda^{2}\right)\right) d \chi^{2} \\
\omega_{D}= & {\left[(1-\lambda)^{2} d\left(w_{1}^{2}\right)+(1+\lambda)^{2} d\left(w_{2}^{2}\right)\right]^{2} } \\
& -\left[\left(1+\lambda^{2}\right)(3-2 \zeta)-2 \lambda\left(w_{1}^{2}-w_{2}^{2}\right)\right]\left[(1-\lambda)^{2} d w_{1}^{2}+(1+\lambda)^{2} d w_{2}^{2}\right] \\
& +8 \lambda(\zeta+1)\left[(1-\lambda)^{2} d w_{1}^{2}-(1+\lambda)^{2} d w_{2}^{2}\right] .
\end{aligned}
$$

Pilch and Warner also computed the near-singularity metric in [20]. Their metric is reproduced by setting $f_{0}=\omega_{\|}=\omega_{D}=0$ and $\frac{1}{\zeta} Y_{v}^{(0,0)} \rightarrow \sigma^{1}$ (modulo a typo in one of the coefficients of $\left.d w_{1}^{2}\right)$. Note that $d \chi^{2}+\frac{1}{4} \chi^{2}\left(\sigma_{1}^{2}+\sigma_{2}^{2}+\sigma_{3}^{2}\right)$ is just the flat metric on $R^{4}$ and the terms in the first line of (5.6) combine to give eight dimensional Minkowski spacetime. This was interpreted in [20] as evidence that the singularity is associated with 7-branes. We cannot however ignore the terms with $f_{0}, \omega_{\|}, \omega_{D}$ and $\frac{1}{\zeta} Y_{v}^{(0,0)}$ because they are of the same order as $\frac{1}{4} \chi^{2}\left(\sigma_{1}^{2}+\sigma_{2}^{2}+\sigma_{3}^{2}\right)$. Taking these terms into account, we find no evidence for 7-branes in the near-singularity structure of the metric. At the position of the $5 d$ singularity, $\chi=0$, the $10 d$ metric is of co-dimension 4 :

$$
d s^{2}=\frac{\zeta^{1 / 4}}{\sqrt{2}}\left(2 e^{2 C_{1}}\left(1-\lambda^{2}\right)^{1 / 3} \eta_{\mu \nu} d x^{\mu} d x^{\nu}+\frac{1}{2 \zeta}\left(\frac{1-\lambda}{1+\lambda} d w_{1}^{2}+\frac{1+\lambda}{1-\lambda} d w_{2}^{2}\right)\right) .
$$

Note that the limit $\chi \rightarrow 0$ is not a decoupling limit, i.e. the metric in (5.6) does not solve the bulk equations of motion and its curvature does not agree with (5.4). To properly account for (5.4) one needs to keep higher order terms in $\chi$. First consider the ten-dimensional metric $G_{M N}$. One can check that in the expansion in the radial coordinate $\chi$ around $\chi=0$ the lowest order in $\chi$ that occurs is the constant order $\chi^{0}$, which is also manifest in (5.6). The same analysis performed on the full inverse metric $G^{M N}$ shows that its lowest order in $\chi$ is the order $\chi^{-2}$. Given this information we can deduce to which orders we need to expand $G_{M N}$ and $G^{M N}$ in order to obtain results consistent with the full computation of the Ricci scalar in the limit $\chi \rightarrow 0$. Schematically the Riemann tensor and the Ricci scalar are given in terms of the metric and its inverse as follows:

$$
\begin{aligned}
R_{M N R S} & \sim \partial^{2} G+G^{-1} \partial G \partial G \\
R & \sim G^{-1} G^{-1}\left(\partial^{2} G+G^{-1} \partial G \partial G\right) .
\end{aligned}
$$

Since $G^{-1} \sim 1 / \chi^{2}$ to get the correct constant term in the Ricci scalar the Riemann tensor needs to be at least of order $\chi^{4}$. Then, from the second term in (5.11) we infer that $\partial G \partial G$ has to be at least of order $\chi^{6}$, and since a derivative with respect to $\chi$ lowers the order in $\chi$ by $1, G$ has to be at least of order $\chi^{7}$. Similarly, one can deduce that one needs to keep terms at least up to order $\chi^{4}$ in the inverse metric $G^{-1}$. We have explicitly checked that keeping the metric and inverse metric to these orders one indeed obtains a curvature scalar consistent with (5.4). 
Similarly, one can study the order to which one has to keep the other fields in order for the bulk equations to be satisfied, order by order in $\chi^{2}$. In general, one cannot truncate this series at some fixed order and have the field equations satisfied, as different orders contribute to different terms in the field equations.

We now provide the near-singularity behaviour of the warp factor and all other fields.

Warp-factor. Following [20] we define

$$
\xi^{2}=\Delta^{-8 / 3}
$$

Then the warp factor has the following leading behaviour as $\chi \rightarrow 0$,

$$
\xi=\frac{8 \zeta^{1 / 2}}{\chi^{4}}+\mathcal{O}\left(\chi^{3}\right)
$$

Axion/dilaton. In the limit $\chi \rightarrow 0$ the axion/dilaton matrix $m_{\alpha \beta}$ is regular and takes the following form:

$$
m_{\alpha \beta}=\zeta^{-1 / 2}\left(\begin{array}{cc}
1+w_{1} & w_{2} \\
w_{2} & 1-w_{1}
\end{array}\right)+\mathcal{O}\left(\chi^{2}\right) .
$$

2-form potential. In the limit $\chi \rightarrow 0$ the 2 -form reduces to the following expression,

$$
\begin{aligned}
& C^{1}=\frac{\sqrt{3}}{\zeta}\left(Y_{v}^{(0,0)} \wedge Y_{v}^{(1,1)}-\frac{1}{2} d w_{1} \wedge Y_{v}^{(1,0)}-\frac{1}{2} d w_{2} \wedge Y_{v}^{(1,1)}\right)+\mathcal{O}\left(\chi^{2}\right) \\
& C^{2}=\frac{\sqrt{3}}{\zeta}\left(-Y_{v}^{(0,0)} \wedge Y_{v}^{(1,1)}+\frac{1}{2} d w_{1} \wedge Y_{v}^{(1,1)}-\frac{1}{2} d w_{2} \wedge Y_{v}^{(1,0)}\right)+\mathcal{O}\left(\chi^{2}\right)
\end{aligned}
$$

where the $\mathrm{SO}(3)_{\text {diag }}$ invariant vector harmonics $Y_{v}^{(k, m)}=Y_{v n}^{(k, m)} d y^{n}$ are 1-forms on the $S^{5}$ cotangent bundle, and $k$ and $m$ are integers that label the harmonics (see appendix D for more details).

4-form potential. The limit $\chi \rightarrow 0$ for the 4 -form is regular and gives the following result

$$
C=\stackrel{\odot}{C}+\frac{1}{32} \frac{w_{2}}{\sqrt{\zeta}\left(1+w_{1}\right)} d w_{1} \wedge d w_{2} \wedge \sigma^{2} \wedge \sigma^{3}+\frac{1}{16}\left(w_{1} d w_{2}-w_{2} d w_{1}\right) \wedge \sigma^{1} \wedge \sigma^{2} \wedge \sigma^{3}+\mathcal{O}\left(\chi^{2}\right)
$$

6-form potential. We get

$$
C_{(6)} \rightarrow \frac{\sqrt{3}}{2} e^{4 C_{1}}\left(1-\lambda^{2}\right)^{2 / 3} d \tau \wedge d x^{1} \wedge d x^{2} \wedge d x^{3} \wedge(\chi d \chi) \wedge\left(\begin{array}{c}
Y_{v}^{(1,1)} \\
-Y_{v}^{(1,0)}
\end{array}\right)+\mathcal{O}\left(\chi^{2}\right)
$$

The coordinate $\tau$ denotes the time. Note that there are powers of $\chi$ that come from the volume form $\omega_{\mu \nu \rho \sigma \tau}$ and cancel some of the divergences. See equation (C.48) for the expansion of $\omega_{\mu \nu \rho \sigma \tau}$ in terms of the radial coordinate. 


\subsection{The $\lambda=1$ case}

We now set $\lambda=1$ first and then take the $\chi \rightarrow 0$ limit. The Ricci scalar becomes

$$
R^{(\lambda=1)}=\frac{1}{6 \sqrt{3}}\left(\frac{8}{\chi^{2}}-1\right) \frac{\left(8+w_{1}^{2}-8 w_{2}^{2}\right)\left(10-w_{1}^{2}-10 w_{2}^{2}\right)}{\left(4-w_{1}^{2}-4 w_{2}^{2}\right)^{9 / 4}}+\mathcal{O}(\chi) .
$$

Thus in this case the $10 d$ metric is still singular at $\chi=0$, though diverging at slower rate than the $5 d$ solution. In addition, the metric is singular at $\left(w_{1}, w_{2}\right)=(0, \pm 1)$ (which corresponds to $(\theta, \phi)=(\pi / 4, \pi / 2 \pm \pi / 2))$, but there is no ring singularity anymore. The metric itself takes the following form

$$
\begin{aligned}
d s_{5}^{2}= & \Omega^{1 / 2}\left[12^{1 / 3} e^{2 C_{1}} \eta_{\mu \nu} d x^{\mu} d x^{\nu} \chi^{2 / 3}+\left(\frac{3}{8} \chi^{2}+1\right) d \chi^{2}+\frac{1}{24 \Omega^{2}}\left(\frac{8}{\chi^{2}}-1\right) d w_{2}^{2}+\frac{\chi^{2}}{4 \zeta} \delta_{2}\right] \\
& +\mathcal{O}\left(\chi^{7 / 3}\right) .
\end{aligned}
$$

The differential $\delta_{2}$ is given by

$$
\begin{aligned}
\delta_{2}= & Y_{v}^{(0,0)^{2}}+\frac{3}{4} d w_{1}^{2}-\frac{1}{4} d w_{2}^{2}-\frac{\zeta}{48 \Omega^{2}} d w_{2}^{2}-\frac{\zeta\left(1+w_{2}^{2}\right)}{18 \Omega^{4}} d w_{2}^{2}+\frac{2-w_{1}^{2}}{6 \Omega^{2}} d w_{2}^{2}+\frac{w_{1} w_{2}}{2 \Omega^{2}} d w_{1} d w_{2} \\
& +\frac{w_{1}}{3 \Omega^{2}} Y_{v}^{(0,0)} d w_{2}+\frac{9}{2+w_{1}} Y_{v}^{(1,0)^{2}}+\frac{2+w_{1}}{\Omega^{2}}\left(\frac{2 w_{2}}{2+w_{1}} Y_{v}^{(1,0)}-Y_{v}^{(1,1)}\right)^{2} .
\end{aligned}
$$

The leading order terms in this metric reproduce the result found by Pilch and Warner [20], but we have additional subleading terms.

Warp-factor. The warp factor has the following leading behaviour under $\lambda \rightarrow 1$, then $\chi \rightarrow 0$

$$
\xi=\frac{16 \hat{\Omega}}{\chi^{4}}+\mathcal{O}\left(\chi^{-3}\right)
$$

where

$$
\hat{\Omega}=\frac{1}{3} \sqrt{4-w_{1}^{2}-4 w_{2}^{2}} .
$$

Axion/dilaton. The axion/dilaton matrix $m_{\alpha \beta}$ is regular and takes the following form:

$$
m_{\alpha \beta}=\frac{1}{3 \hat{\Omega}}\left(\begin{array}{cc}
2+w_{1} & 2 w_{2} \\
2 w_{2} & 2-w_{1}
\end{array}\right)+\mathcal{O}\left(\chi^{2}\right) .
$$

2-form potential. The limit of the 2 -form may be written in terms of wedge products of the vector harmonics found in appendix D:

$$
\begin{aligned}
& C^{1}=\frac{\sqrt{3}}{\zeta}\left(Y_{v}^{(0,0)}-\frac{2 \zeta-3 w_{1}}{18 \hat{\Omega}^{2}} d w_{2}\right) \wedge Y_{v}^{(1,1)}-\frac{\sqrt{3}}{2 \zeta}\left(d w_{1}+\frac{w_{1} w_{2}}{3 \hat{\Omega}^{2}}\right) \wedge Y_{v}^{(1,0)}+\mathcal{O}\left(\chi^{2}\right) \\
& C^{2}=\frac{\sqrt{3}}{\zeta}\left(-Y_{v}^{(0,0)}-\frac{2 \zeta+3 w_{1}}{18 \hat{\Omega}^{2}} d w_{2}\right) \wedge Y_{v}^{(1,0)}+\frac{\sqrt{3}}{2 \zeta}\left(d w_{1}+\frac{w_{1} w_{2}}{3 \hat{\Omega}^{2}}\right) \wedge Y_{v}^{(1,1)}+\mathcal{O}\left(\chi^{2}\right)
\end{aligned}
$$


4-form potential. The limit of the 4-form is regular, and is given by

$$
\begin{aligned}
C= & \stackrel{\circ}{C}+\frac{w_{2} \zeta^{1 / 2}}{72\left(1+w_{1}\right) \hat{\Omega}^{2}} d w_{1} \wedge d w_{2} \wedge \sigma^{2} \wedge \sigma^{3}-\frac{w_{1}}{48 \hat{\Omega}^{2}} d w_{2} \wedge \sigma^{1} \wedge \sigma^{2} \wedge \sigma^{3} \\
& +\frac{1}{16}\left(w_{1} d w_{2}-w_{2} d w_{1}\right) \wedge \sigma^{1} \wedge \sigma^{2} \wedge \sigma^{3}+\mathcal{O}\left(\chi^{2}\right) \\
= & \stackrel{C}{C}+\left(\frac{1+w_{1}}{2}\right)^{-1} \frac{w_{2} \zeta^{1 / 2}}{144 \hat{\Omega}^{2}} d w_{1} \wedge d w_{2} \wedge \sigma^{2} \wedge \sigma^{3}-\frac{w_{1}}{48 \hat{\Omega}^{2}} d w_{2} \wedge \sigma^{1} \wedge \sigma^{2} \wedge \sigma^{3} \\
& +\frac{1}{16}\left(w_{1} d w_{2}-w_{2} d w_{1}\right) \wedge \sigma^{1} \wedge \sigma^{2} \wedge \sigma^{3}+\mathcal{O}\left(\chi^{2}\right)
\end{aligned}
$$

6-form potential. For the 6 -form we get

$$
C_{(6)} \rightarrow-\frac{7 e^{4 C_{1}}}{2^{2 / 3} 3^{5 / 6}} d \tau \wedge d x^{1} \wedge d x^{2} \wedge d x^{3} \wedge\left(\chi^{7 / 3} d \chi\right) \wedge\left(\begin{array}{c}
Y_{v}^{(1,1)} \\
-Y_{v}^{(1,0)}
\end{array}\right)+\mathcal{O}(\chi)^{10 / 3}
$$

where $\tau$ is the time coordinate. Again, as in the case $\lambda<1$, there are some powers of $\chi$ that come from the volume form $\omega_{\mu \nu \rho \sigma \tau}$ and cancel some divergences. See equation (C.48) for the expansion of $\omega_{\mu \nu \rho \sigma \tau}$ in terms of the radial coordinate.

\subsection{Different frames}

Since the solution involves non-trivial scalars there is an intrinsic ambiguity in the definition of the spacetime metric: one can rescale the metric with powers of the scalars. Different probe behaviours see different metrics and different conformal frames carry different physical meaning. For example, supergravity probes see the Einstein frame metric and strings see the string frame metric. In some cases singular geometries are regular in a different frame. For example, the geometry of non-conformal $\mathrm{D} p$ branes is singular in the Einstein and string frame but it is regular in the "dual frame" [35] and this is also the frame best suited for holography $[36,37]$. Here we want to analyse the dependence of the singularity on the choice of frame.

Usually one uses the dilaton when discussing different frames. ${ }^{14}$ Since our solution has both an axion and a dilaton we will explore a general rescaling by both: $g_{\hat{\mu} \hat{\nu}} \rightarrow \tilde{g}_{\hat{\mu} \hat{\nu}}=\Omega^{2} g_{\hat{\mu} \hat{\nu}}$ with the scaling factor $\Omega=e^{x \phi} C_{0}^{z}$ given by some powers of the dilaton $e^{\phi}$ and the axion $C_{0}$ parameterised through constants $x$ and $z$. Given the definition of the axion/dilaton matrix $m_{\alpha \beta}$

$$
m_{\alpha \beta}=\frac{1}{\Im \tau}\left(\begin{array}{cc}
|\tau|^{2} & -\Re \tau \\
-\Re \tau & 1
\end{array}\right), \quad \tau=C_{0}+i e^{-\Phi},
$$

we can write the rescaling parameter as $\Omega=m_{22}^{x}\left(-m_{12} / m_{22}\right)^{z}$. To compute the effect of rescaling on the Ricci scalar we can use the standard formula for the Weyl rescaling of the Ricci scalar (see for example [38]):

$$
\tilde{R}=\Omega^{-2}\left[R-18 g^{\hat{\mu} \hat{\nu}} \nabla_{\hat{\mu}} \nabla_{\hat{\nu}} \log \Omega-72 g^{\hat{\mu} \hat{\nu}}\left(\nabla_{\hat{\mu}} \log \Omega\right)\left(\nabla_{\hat{\nu}} \log \Omega\right)\right] .
$$

\footnotetext{
${ }^{14}$ One reason for this is that the axion is more properly viewed as a 0 -form potential and has an associated gauge invariance.
} 
The $\lambda<1$ case. After the rescaling the Ricci scalar takes the following form:

$$
\tilde{R}^{(\lambda<1)}=\frac{\mathcal{P}^{(\lambda<1)}\left(w_{1}, w_{2}\right)}{\left(1-w_{1}^{2}-w_{2}^{2}\right)^{\frac{5}{4}-x} w_{2}^{2+2 z}\left(1-w_{1}\right)^{2+2 x-2 z}}+\mathcal{O}(\chi),
$$

where $\mathcal{P}^{(\lambda<1)}\left(w_{1}, w_{2}\right)$ is a polynomial in $w_{1}$ and $w_{2}$ with coefficients containing $x, z$, and $\lambda$. After a careful inspection it is evident that there is no choice of $x$ and $z$ that removes the denominator. One can also show that the numerator $\mathcal{P}^{(\lambda<1)}\left(w_{1}, w_{2}\right)$ is non-zero for any choice of $x, z$, and $\lambda<1$, therefore the singularity in the curvature cannot be completely removed.

One can now study what type of singular behaviour the terms in the denominator entail. The term $\left(1-w_{1}^{2}-w_{2}^{2}\right)$ is just the original ring singularity along the circle $w_{1}^{2}+w_{2}^{2}=$ 1. The term $w_{2}$ leads to singularities on parts of the ring corresponding to $\theta \in\{0, \pi\}$ or $\phi=\pi / 2$, while the term $\left(1-w_{1}\right)$ reduces the singularity to a single point $\left(w_{1}, w_{2}\right)=(1,0)$, which is equivalent to the value $\theta=0$. Thus we see that the least singular behaviour that we can get is achieved by choosing $x \geq 5 / 4$ and $z \leq-1$ which leads to a singularity of type $\left(1-w_{1}\right)^{a}$ with $a \geq 9 / 4$, i.e. in this case we only have a singularity at a single point. It would be interesting to understand the meaning of these frames.

The $\lambda=1$ case. We can now repeat the same analysis for the case $\lambda=1$. The transformed Ricci scalar has the form

$$
\tilde{R}^{(\lambda=1)}=\left(\frac{8}{\chi^{2}}-1\right) \frac{\mathcal{P}^{(\lambda=1)}\left(w_{1}, w_{2}\right)}{\left(4-w_{1}^{2}-4 w_{2}^{2}\right)^{\frac{9}{4}-x} w_{2}^{2 z}\left(2-w_{1}\right)^{2+2 x-2 z}}+\mathcal{O}(\chi) .
$$

First of all, also in this case it can be shown that $\mathcal{P}^{(\lambda=1)}\left(w_{1}, w_{2}\right)$ cannot be identically zero for any choice of $x$ and $z$. This means that the singularity $\chi^{-2}$ in the radial coordinate can never be removed. Notice however that the term $\left(2-w_{1}\right)$ in the denominator is never zero since $-1 \leq w_{1} \leq 1$, and therefore we can arrange that the singularity in the angular directions is removed completely by choosing $x \geq 9 / 4$ and $z \leq 0$.

\section{Conclusions and outlook}

In this paper we presented the uplift of the GPPZ solution to ten dimensions. The original GPPZ solution involved two real scalars, which correspond to the norm of a $\mathcal{N}=1$ supersymmetric mass deformation of $\mathcal{N}=4 \mathrm{SYM}$ (which is a complex parameter) and the norm the gaugino bilinear. The complex scalars corresponding to the sources that couple to the complex QFT operators are part of a consistent truncation of $D=5$ maximal supergravity containing, besides the two complex scalars, the metric and a $\mathrm{U}(1)$ gauge field, which is dual to the $\mathrm{U}(1)_{R}$ current. We generalised the GPPZ solution to complex fields, which either have constant phases or a spacetime dependent but U(1)-covariantly constant phase. In ten dimensions the solution with non-zero phases can be obtained from the one with no phases by a combination of coordinate transformation on $S^{5}$ and an $\mathrm{SO}(2)$ rotation in $\mathrm{SL}(2, R)$ that correspond to a $\mathrm{U}(1)_{R}$ transformation and the bonus $\mathrm{U}(1)$ transformation on the QFT side, respectively. The ten-dimensional solution has an infrared singularity 
whose structure depends on the ratio of the mass deformation parameter and the gaugino condensate, which we denoted by $\lambda$. It was argued in [6] that the singularity is acceptable only if $\lambda \leq 1$. In the $\lambda<1$ case, the non-compact part of the metric is regular and there is only a ring singularity in the $S^{5}$ part. In the $\lambda=1$ case there is a singularity both in the radial direction (but milder than that of the five dimensional solution) and also at two points on $S^{5}$. Intriguingly, one can find conformal frames such that there is only a singularity in the Ricci scalar at one point in $S^{5}$ in the case of $\lambda<1$, or only in the radial coordinate in the case of $\lambda=1$. We note however that these comments are based on the Ricci scalar, and we have not checked the behaviour of other curvature invariants.

The metric and axion-dilaton field of the solution with zero phases agree exactly with those of the a partial uplift by Pilch and Warner and the singularity structure agrees with their findings. However we find additional subleading terms in the near-singularity metric, which do not fit in the interpretation of the singularity as due to seven-branes.

We also compared the asymptotics of the uplifted solution with that of the PolchinskiStrassler solution [5] and the zero temperature limit of the Freedman-Minahan solution [31]. Our results agree with that of Freedman-Minahan and disagree with Polchinski-Strassler. We have checked that we use the same boundary condition with Polchinski-Strassler, i.e. the non-normalizable modes (sources) are in exact agreement: the metric is asymptotically $A d S_{5} \times S^{5}$ and the part of the 3 -form field strength that depends on the deformation parameter are in exact agreement. The subleading terms however disagree. Recalling that the subleading terms (up to the order where the vevs appear) are uniquely fixed in terms of the sources by the field equations [28], we infer that the Polchinski-Strassler 10d fields do not satisfy the IIB field equations near the boundary of AdS.

There are still many things to do. The most urgent question is whether the solution in sourced by branes. While we have checked explicitly with Mathematica that the uplift solution solves the IIB equations, there may be delta-function sources in the field equations coming from the couplings of IIB fields to the worldvolume of branes. In principle, one can detect such terms by a careful analysis of the way the field equations are satisfied. To illustrate this point suppose that while checking the field equations one needs to evaluate

$$
\square \frac{1}{r^{n-2}}
$$

where $\square$ is the Laplacian in $n$ (Euclidean) dimensions and $r$ is the distance from the origin. This term is proportional to a delta function and hunting for such terms should provide the delta function sources. However, given the complexity of the uplifted solution and of the IIB field equations and the fact that we do not know the positions and orientations of the branes (if present at all), this is a rather daunting task.

One way to proceed is to observe that one may check that (6.1) has indeed a delta function by multiplying it with a smooth test function and integrating over all space: if there is a source then the answer for the integral will be non-zero, otherwise it will be zero. We have devised a version of this method to check whether the uplifted solution is supported by branes or not. The analysis is complicated by the fact that the bulk solution has a (conformal) boundary and it is singular, and thus one needs to carefully disentangle 
the three possible contributions to the integral: due to the boundary, from the singularity and due to delta functions. We will report on this analysis elsewhere.

To understand the implications of the singularity it will also be useful to analyze how different probes respond when placed in this background. Given that the solution is very explicit it should be straightforward to perform such analysis.

It is also interesting to understand to which of the many $\mathcal{N}=1^{*}$ vacua the GPPZ flow and the uplifted solution correspond to. Now that we have the solution in ten dimensions one may proceed to extract the 1-point functions of operators other than the gaugino bilinear. This can be done following the framework of Kaluza-Klein holography [29] and it should allow us to definitely establish whether this solution is dual to the confining vacuum of $\mathcal{N}=1^{*}$, a different vacuum of this theory, or that it is unphysical (because of the singularity) and it should be discarded. We hope to return to this and related issues in the near future.

Finally, it is natural to apply the uplift techniques to other five-dimensional solutions, as, for instance, the solution of $\mathcal{N}=8$ gauge supergravity dual to the $\mathcal{N}=2^{*}$ theory, a deformation of $\mathcal{N}=4$ SYM by a mass for two of the chiral multiplets [33, 39-41]. Recently the approach of consistent truncation has been used to compute the ten-dimensional solution dual to $\mathcal{N}=2^{*}$ on $S^{4}$ [42]. It would be interesting to study other cases, with less supersymmetry or different compact manifolds.

Note added. Soon after this paper appeared in the arXiv, the paper [43] appeared which has some overlap with our results.

\section{Acknowledgments}

We acknowledge the hospitality of the Galileo Galilei Institute for Theoretical Physics, where this work was initiated during the workshop "Supergravity: what next?". KS is supported in part by the Science and Technology Facilities Council Consolidated Grant "New Frontiers in Particle Physics and Cosmology", ST/P000711/1. This project has received funding from the European Union's Horizon 2020 research and innovation programme under the Marie Sklodowska-Curie grant agreement No 690575. SS acknowledges the funding from the Mayflower Scholarship from the University of Southampton. MP is partly supported by ILP LABEX (ANR-10-LABX-63) and the Idex SUPER (ANR-11IDEX-0004-02).

\section{A IIB uplift of $D=5$ maximal supergravity}

$D=5$ maximal $\mathrm{SO}(6)$ gauged supergravity $[7,8,34]$ is a consistent truncation of IIB supergravity around $\mathrm{AdS}_{5} \times S^{5}$. Its field content comprises the $D=5$ metric $g_{\mu \nu}$ together with 42 scalar fields parametrizing a $27 \times 27$ symmetric $\mathrm{E}_{6(6)}$ matrix which we parametrize in an $\mathrm{SL}(6) \times \mathrm{SL}(2)$ basis as

$$
M_{M N}=\left(\begin{array}{ll}
M_{a b, c d} & M_{a b}^{c \beta} \\
M_{c d}^{a \alpha} & M^{a \alpha, c \beta}
\end{array}\right), \quad \text { with inverse } M^{M N}=\left(\begin{array}{cc}
M^{a b, c d} & M^{a b}{ }_{c \beta} \\
M_{a \alpha} c d & M_{a \alpha, c \beta}
\end{array}\right)
$$


according to the decomposition of the fundamental representation of $\mathrm{E}_{6(6)}$ as

$$
\mathbf{2 7} \longrightarrow(\mathbf{1 5}, \mathbf{1}) \oplus\left(\mathbf{6}^{\prime}, \mathbf{2}\right),
$$

under $\mathrm{SL}(6) \times \mathrm{SL}(2)$. Indices $a, b, c, d=1, \ldots, 6$, and $\alpha, \beta=1,2$, label the fundamental representations of $\mathrm{SL}(6)$ and $\mathrm{SL}(2)$, respectively. Index pairs $a b$ and $c d$ in (A.1) are antisymmetric. The remaining bosonic field content in five dimensions is given by 15 nonabelian vectors fields $A_{\mu}^{a b}$ and 12 topologically massive two-forms $B_{\mu \nu, a \alpha}$. The truncation we are eventually interested in and which carries the GPPZ solution [6] carries four scalar fields, a single vector and no two-forms.

In this section we collect the relevant IIB uplift formulae of $D=5$ supergravity from [9] (see also [40, 44-47]), in the next section we explicitly evaluate these formulas for the fourscalar truncation. The IIB fields are expressed in terms of the $D=5$ fields introduced above while their dependence on the five internal coordinates $y^{m}$ is carried by the fundamental $S^{5}$ sphere harmonics, $\mathcal{Y}^{a},(a=1, \ldots, 6)$, with $\mathcal{Y}^{a} \mathcal{Y}^{a}=1$, and the $S^{5}$ Killing vectors

$$
\mathcal{K}_{[a b] m}=-\sqrt{2} \mathcal{Y}^{[a} \partial_{m} \mathcal{Y}^{b]}, \quad m=1, \ldots, 5 .
$$

By $\stackrel{\circ}{G}_{m n}$ we denote the round metric on $S^{5}$ which can be expressed as

$$
\stackrel{\circ}{G}_{m n}=\mathcal{K}_{[a b] m} \mathcal{K}_{[a b] n},
$$

in terms of the Killing vectors (A.3). We also define its volume form

$$
\left.\stackrel{\circ}{\omega}_{k l m n p} \equiv \sqrt{\operatorname{det} \stackrel{\circ}{G}} \varepsilon_{k l m n p} \equiv 5 \partial_{[k} \stackrel{\circ}{l m n p}\right],
$$

in terms of a 4 -form potential $\stackrel{C}{k l m n}_{k}$. We will also need the tensors

$$
\begin{aligned}
\mathcal{K}_{[a b] m n} & \equiv \partial_{m} \mathcal{K}_{[a b] n}-\partial_{n} \mathcal{K}_{[a b] m}, \\
\mathcal{K}_{[a b] k l m} & \equiv \frac{1}{2} \stackrel{\circ}{\text { klmnp }} \mathcal{K}_{[a b]}{ }^{n p},
\end{aligned}
$$

where indices $n, p$ on the r.h.s. are raised with the background metric (A.4).

In terms of these objects, the IIB metric takes the following form

$$
\begin{aligned}
d s^{2}= & \Delta^{-2 / 3}(x, y) g_{\mu \nu}(x) d x^{\mu} d x^{\nu} \\
& +G_{m n}(x, y)\left(d y^{m}+\mathcal{K}_{[a b]}{ }^{m}(y) A_{\mu}^{a b}(x) d x^{\mu}\right)\left(d y^{n}+\mathcal{K}_{[c d]}^{n}(y) A_{\nu}^{c d}(x) d x^{\nu}\right),
\end{aligned}
$$

with the internal block $G_{m n}(x, y)$ given by inverting the matrix

$$
\mathcal{G}^{m n}(x, y)=\Delta(x, y)^{2 / 3} \mathcal{K}_{[a b]}^{m}(y) \mathcal{K}_{[c d]}^{n}(y) M^{a b, c d}(x),
$$

in terms of the submatrix $M^{a b, c d}(x)$ from (A.1). We use indices $\mu, \nu$ and $m, n$ for the external five and internal five coordinates, respectively. The warp factor $\Delta(x, y)$ is defined as

$$
\Delta(x, y)=\frac{\left(\operatorname{det} G_{m n}(x, y)\right)^{1 / 2}}{\left(\operatorname{det} \stackrel{\circ}{G}_{m n}(y)\right)^{1 / 2}} .
$$


The IIB dilaton and axion combine into a symmetric $\mathrm{SL}(2)$ matrix $m_{\alpha \beta}$ whose inverse is given by

$$
m^{\alpha \beta}(x, y)=\Delta(x, y)^{4 / 3} \mathcal{Y}^{a}(y) \mathcal{Y}^{b}(y) M^{a \alpha, b \beta}(x) .
$$

The relevant components of the IIB 2-form doublet and 4-form gauge potentials are given by

$$
\begin{aligned}
& C_{m n}{ }^{\alpha}=-\frac{1}{2} \varepsilon^{\alpha \beta} \Delta^{4 / 3} m_{\beta \gamma} \mathcal{Y}^{c} \mathcal{K}_{[a b] m n} M_{a b}{ }^{c \gamma}, \\
& C_{\mu k m n}=-\frac{1}{8} \stackrel{\circ}{\omega}_{k m n p q} \stackrel{\circ}{\nabla}^{p} \mathcal{K}_{[a b]}^{q} A_{\mu}^{a b}-\mathcal{K}_{[a b]}^{p} A_{\mu}{ }^{[a b]} \stackrel{\circ}{C}_{p k m n} \\
& C_{m \mu \nu \rho}=-\frac{1}{32} \mathcal{K}_{[a b] m}\left(2 \sqrt{|g|} \varepsilon_{\mu \nu \rho \sigma \tau} M_{a b, c d} F^{\sigma \tau c d}+3 \sqrt{2} \varepsilon_{a b c d e f} \partial_{[\mu} A_{\nu}{ }^{c d} A_{\rho]}{ }^{e f}\right), \\
& C_{k l m n}=\stackrel{\circ}{C}_{k l m n}-\frac{1}{6} \stackrel{\circ}{\omega}_{k l m n p} \stackrel{\circ}{G}^{p q} \Delta^{-1} \partial_{q} \Delta, \\
& C_{\mu \nu \rho \sigma}=-\frac{1}{16} \mathcal{Y}^{a} \mathcal{Y}^{b} \sqrt{|g|} \varepsilon_{\mu \nu \rho \sigma \tau} D^{\tau} M_{b c, N} M^{N c a}+\Lambda_{\mu \nu \rho \sigma},
\end{aligned}
$$

with $F_{\mu \nu}^{a b}$ the five-dimensional field strength of $A_{\mu}{ }^{a b}$. The function $\Lambda_{\mu \nu \rho \sigma}(x)$ in the last line is defined by integrating

$$
\begin{aligned}
D_{[\mu} \Lambda_{\nu \rho \sigma \tau]}= & \frac{1}{600} \sqrt{|g|} \varepsilon_{\mu \nu \rho \sigma \tau}\left(10 \delta_{h}^{d} \delta_{e}^{a}+2 M^{f d, g a} M_{g h, f e}-M_{e \alpha}{ }^{g a} M_{g h}{ }^{d \alpha}\right) M^{b h, e c} \delta_{c d} \delta_{a b} \\
& -\frac{1}{480} \sqrt{|g|} \varepsilon_{\mu \nu \rho \sigma \tau} D_{\lambda}\left(M^{N a c} D^{\lambda} M_{a c, N}\right) \\
& +\frac{1}{240} \sqrt{|g|} \varepsilon_{\mu \nu \rho \sigma \tau} M_{a b, c d} F_{\kappa \lambda}^{a b} F^{\kappa \lambda c d}+\frac{1}{32} \sqrt{2} \varepsilon_{a b c d e f} F_{[\mu \nu}^{a b} F_{\rho \sigma}{ }^{c d} A_{\tau]}{ }^{e f} .
\end{aligned}
$$

The $p$-forms (A.11) are given in the standard Kaluza-Klein basis

$$
D y^{m}=d y^{m}+\mathcal{K}_{[a b]}^{m}(y) A_{\mu}^{a b}(x) d x^{\mu},
$$

cf. (A.7). As compared to the full uplift formulas [9] we have suppressed in the $p$ forms (A.11) all terms anti-symmetric in more than one vector field since these will not survive in the truncation to a single vector field which is of interest here.

\section{B Parametrization of the scalar matrix}

In this appendix we spell out the explicit parametrization of the scalar $E_{6(6)}$ matrix $M_{M N}$ (A.1) in the 4-scalar truncation of $D=5$ maximal supergravity. To this end, we go to a complex basis, in which the $\mathrm{SL}(6)$ vector decomposes according to

$$
\left\{X_{a}\right\} \longrightarrow\left\{X_{i}, X_{\bar{i}}=X_{i}^{*}\right\}, \quad i, \bar{i}=1,2,3 .
$$

In this decomposition, the $\mathrm{E}_{6(6)}$ matrix (A.1) decomposes as

$$
M_{M N}=\left(\begin{array}{lllll}
M_{i j, k l} & M_{i j, k \bar{l}} & M_{i j, \overline{k l}} & M_{i j}^{k \beta} & M_{i j}{ }_{\bar{k} \beta} \\
M_{i \bar{j}, k l} & M_{i \bar{j}, k \bar{l}} & M_{i \bar{j}, \overline{k l}} & M_{i \bar{j}}^{k \beta} & M_{i \bar{j}} \bar{k} \\
M_{\overline{i j}, k l} & M_{\overline{i j}, k \bar{l}} & M_{\overline{i j}, \overline{k l}} & M_{\overline{i j}}^{k \beta} & M_{\overline{i j}}{ }_{\bar{k} \beta} \\
M^{i \alpha}{ }_{k l} & M^{i \alpha}{ }_{k \bar{l}} & M^{i \alpha} \overline{k l} & M^{i \alpha, k \beta} & M^{i \alpha, \bar{k} \beta} \\
M^{\bar{i} \alpha}{ }_{k l} & M^{\bar{i} \alpha}{ }_{k \bar{l}} & M^{\bar{i} \alpha}{ }_{\overline{k l}} & M^{\overline{i \alpha}, k \beta} & M^{\overline{i \alpha}, \bar{k} \beta}
\end{array}\right),
$$


with its non-vanishing entries given in terms of the $\mathrm{SL}(2)$ vector $v^{\alpha} \equiv(1,-i)^{\alpha}$ by

$$
\begin{aligned}
M_{i j, k l} & =-\delta_{i j}^{k l} e^{-i(\varphi+\omega)} \sinh \left(\frac{2 m}{\sqrt{3}}\right) \sinh (2 \sigma) \\
M_{i j, \overline{k l}} & =\delta_{i j}^{\overline{k l}} \cosh \left(\frac{2 m}{\sqrt{3}}\right) \cosh (2 \sigma) \\
M_{i j}{ }^{k \alpha} & =\frac{1}{2} \varepsilon^{i j k} e^{-i \varphi} \sinh \left(\frac{2 m}{\sqrt{3}}\right) \cosh (2 \sigma) v^{\alpha} \\
M_{\overline{i j}}^{k \alpha} & =-\frac{1}{2} \varepsilon^{i j k} e^{i \omega} \cosh \left(\frac{2 m}{\sqrt{3}}\right) \sinh (2 \sigma) v^{\alpha}, \\
M_{i \bar{j}} \bar{k}^{\bar{k}} & =\frac{1}{4} \varepsilon^{i j k} e^{-i \varphi} \sinh \left(\frac{4 m}{\sqrt{3}}\right) v^{\alpha} \\
M^{i \alpha, j \beta} & =\frac{1}{2} e^{2 i \varphi} \sinh ^{2}\left(\frac{2 m}{\sqrt{3}}\right)\left(v^{\alpha} v^{\beta}\right)^{*}-\frac{1}{2} e^{i(\omega-\varphi)} \sinh \left(\frac{2 m}{\sqrt{3}}\right) \sinh (2 \sigma) v^{\alpha} v^{\beta}, \\
M^{i \alpha, \bar{j} \beta} & =\frac{1}{2} \cosh ^{2}\left(\frac{2 m}{\sqrt{3}}\right)\left(v^{\alpha}\right)^{*} v^{\beta}+\frac{1}{2} \cosh \left(\frac{2 m}{\sqrt{3}}\right) \cosh (2 \sigma) v^{\alpha}\left(v^{\beta}\right)^{*},
\end{aligned}
$$

together with those components related by complex conjugation. Plugging this explicit form of the scalar matrix into the uplift formulas of appendix A yields the IIB uplift of the 4 -scalar truncation of the $D=5$ theory which we describe in section 3 .

\section{Uplift in Pilch-Warner coordinates}

In this appendix we present the uplift solution in the coordinates introduced in [20]. First let us recall the definition of the new radial coordinate $t$ and other constants

$$
t=e^{-\left(y-C_{1}\right)}, \quad \lambda=e^{3\left(C_{2}-C_{1}\right)}, \quad C_{1}=\log \left(\frac{m_{0}}{\sqrt{3}}\right), \quad C_{2}=\frac{1}{3} \log \left(\frac{\sigma_{0}}{2}\right) .
$$

where $C_{1}$ and $C_{2}$ are the $5 d$ integration constants and $m_{0}, \sigma_{0}$ are related to the leading asymptotic behaviour of the $5 d$ fields $m$ and $\sigma$ (note that $m_{0}, \sigma_{0}$ differ by constants relative to the ones in [20]). Defining $\mu, \nu$ as in (3.13), the solution of the first order equation in terms of these variables takes the form

$$
\mu(t)=\sqrt{\frac{1+\lambda t^{3}}{1-\lambda t^{3}}}, \quad \nu(t)=\sqrt{\frac{1+t}{1-t}} .
$$

Yet another definition of the radial coordinate introduced in [20] is the following

$$
\chi=2(1-t)^{1 / 2}
$$

For the internal manifold we think of the round sphere as embedded in $\mathbb{R}^{6}$ described by the coordinates $y_{1}, \ldots y_{6}$, so that on the sphere $\vec{y}^{2}=1$. The six coordinates can be thought of as split into two triplets

$$
\vec{y}=\left(y_{1}, \ldots y_{6}\right) \rightarrow\left(u_{1}, u_{2}, u_{3}, v_{1}, v_{2}, v_{3}\right) \equiv(\vec{u}, \vec{v}) .
$$


The diagonal $\mathrm{SO}(3)$ acts on $u^{i}$ and $v^{j}$ simultaneously in the vector representation. In [20] the authors show that $\vec{u}$ and $\vec{v}$ can be written as

$$
\begin{aligned}
& \vec{u}=R\left(\begin{array}{c}
0 \\
0 \\
\cos \theta
\end{array}\right) \equiv R \cdot \vec{u}_{0} \\
& \vec{v}=R\left(\begin{array}{c}
0 \\
\sin \theta \sin \phi \\
\sin \theta \cos \phi
\end{array}\right) \equiv R \cdot \vec{v}_{0}
\end{aligned}
$$

with $\theta \in[0, \pi / 2], \phi \in[0, \pi]$ and $R=R\left(\alpha_{1}, \alpha_{2}, \alpha_{3}\right)$ a generic $\mathrm{SO}(3)$ matrix parametrized by three Euler angles $\alpha_{i}$. Following [20] we further define

$$
\begin{aligned}
& w_{1}=2 \vec{u}^{2}-1=\cos (2 \theta) \\
& w_{2}=2 \vec{u} \cdot \vec{v}=\sin (2 \theta) \cos \phi
\end{aligned}
$$

such that the internal manifold is described by the coordinates $\left\{\alpha_{1}, \alpha_{2}, \alpha_{3}, w_{1}, w_{2}\right\}$.

To write form-fields in terms of these coordinates it is useful to compute the differentials $d u^{i}$ and $d v^{i}$. In the coordinates just introduced this translates to

$$
d \vec{u}=d R \cdot \vec{u}_{0}+R \cdot d \vec{u}_{0}=R\left(R^{-1} d R \cdot \vec{u}_{0}+d \vec{u}_{0}\right) .
$$

The Maurer-Cartan form $R^{-1} d R$ can be decomposed into left-invariant 1-forms $\sigma^{i}$, which we list below in (C.13) so that the differential $d u^{i}$ can be written as

$$
d \vec{u}=R\left(i \sigma^{i} T^{i} \cdot \vec{u}_{0}+d \vec{u}_{0}\right)
$$

and analogously for $d v^{i}$. Since in the quantities we are interested in the $\mathrm{SO}(3)$ indices are always contracted the overall factor of $R$ in the differentials drops out. Thus (using the fact that $\left(T^{j}\right)^{i k}=i \epsilon^{i j k}$ for $\left.\mathrm{SO}(3)\right)$ in $\mathrm{SO}(3)$-invariant expressions we can substitute

$$
\begin{aligned}
& d u^{i} \rightarrow \epsilon^{i j k} u_{0}^{j} \sigma^{k}+d u_{0}^{i} \\
& d v^{i} \rightarrow \epsilon^{i j k} v_{0}^{j} \sigma^{k}+d v_{0}^{i} .
\end{aligned}
$$

A particular choice of the $\mathrm{SO}(3)$ rotation matrix $R\left(\alpha_{1}, \alpha_{2}, \alpha_{3}\right)$ leads to the left invariant forms given by

$$
\begin{aligned}
\sigma^{1} & =\cos \alpha_{1} d \alpha_{2}+\sin \alpha_{1} \sin \alpha_{2} d \alpha_{3} \\
\sigma^{2} & =\sin \alpha_{1} d \alpha_{2}-\cos \alpha_{1} \sin \alpha_{2} d \alpha_{3} \\
\sigma^{3} & =d \alpha_{1}+\cos \alpha_{2} d \alpha_{3} .
\end{aligned}
$$

The ranges for the Euler angles are $\alpha_{1}, \alpha_{3} \in[0,2 \pi]$, and $\alpha_{2} \in[0, \pi]$. One may check directly that these forms indeed satisfy

$$
d \sigma^{a}=\frac{1}{2} \epsilon^{a b c} \sigma^{b} \wedge \sigma^{c}
$$


The following variables (also introduced in [20]) are also useful,

$$
\begin{aligned}
\zeta & =1-w_{1}^{2}-w_{2}^{2} \\
\hat{\Omega} & =\frac{1}{3} \sqrt{4\left(1-w_{1}^{2}-w_{2}^{2}\right)+3 w_{1}^{2}}=\frac{1}{3} \sqrt{4-w_{1}^{2}-4 w_{2}^{2}},
\end{aligned}
$$

These variables are the coefficients of the leading terms of the warp factor in the $t \rightarrow 1$ and $\lambda \rightarrow 1, t \rightarrow 1$ limits.

We are now ready to present the solution in terms of these variables.

Warp-factor. The warp-factor $\Delta$ we used earlier and the warp factor $\xi$ in [20] are related by

$$
\xi^{2}=\Delta^{-8 / 3} .
$$

In the new coordinates we find

$$
\begin{aligned}
\xi^{2}= & \frac{1}{\left(1-t^{2}\right)^{4}\left(1-\lambda^{2} t^{6}\right)^{2}} \\
& \times\left[\left(1+t^{2}\right)^{2}\left(1-\lambda^{2} t^{8}\right)^{2}-4 w_{1}^{2} t^{4}\left(1-\lambda t^{2}\right)^{2}\left(1+\lambda t^{4}\right)^{2}-4 w_{2}^{2} t^{4}\left(1+\lambda t^{2}\right)^{2}\left(1-\lambda t^{4}\right)^{2}\right]
\end{aligned}
$$

Metric. The uplifted ten-dimensional metric was already obtained by Pilch and Warner [20], see equations (6.1)-(6.7) in their text. It takes a block form containing the AdS and the $S^{5}$ parts as follows

$$
\begin{aligned}
d s_{10}^{2}= & \xi^{1 / 2} d s_{1,4}^{2}+\xi^{-3 / 2} d s_{5}^{2} \\
d s_{1,4}^{2}= & e^{2 \phi(y)} \eta_{\mu \nu} d x^{\mu} d x^{\nu}+d y^{2} \\
d s_{5}^{2}= & a_{1} d u^{i} d u^{i}+2 a_{2} d u^{i} d v^{i}+a_{3} d v^{i} d v^{i} \\
& +a_{4}\left(u^{i} d v^{i}+v^{i} d u^{i}\right)^{2}+2 a_{5}\left(u^{i} d v^{i}\right)\left(v^{j} d u^{j}\right)+2 a_{6}\left(u^{i} d u^{i}\right)\left(v^{j} d v^{j}\right) .
\end{aligned}
$$

The coefficients $a_{i}$ of the internal metric can be found in equation (6.3) in the Pilch and Warner text [20]. We can expand the fields $\mu(t)$ and $\nu(t)$ in terms of the radial coordinate $t$ to get the following expressions for the coefficients $a_{i}$ :

$$
\begin{aligned}
& a_{1}=\frac{\left(1+\lambda t^{4}\right)\left(1+t^{2}\left(1-2 w_{1}\right)\left(1-\lambda t^{2}\right)-\lambda t^{6}\right)}{\left(1-t^{2}\right)^{2}\left(1-\lambda^{2} t^{6}\right)} \\
& a_{2}=\frac{-2 w_{2} t^{2}\left(1+\lambda t^{2}\right)\left(1-\lambda t^{4}\right)}{\left(1-t^{2}\right)^{2}\left(1-\lambda^{2} t^{6}\right)} \\
& a_{3}=\frac{\left(1+\lambda t^{4}\right)\left(1+t^{2}\left(1+2 w_{1}\right)\left(1-\lambda t^{2}\right)-\lambda t^{6}\right)}{\left(1-t^{2}\right)^{2}\left(1-\lambda^{2} t^{6}\right)} \\
& a_{4}=\frac{t^{2}\left(1+\lambda t^{2}\right)^{2}\left(1+\lambda t^{4}\right)\left(1+3 t^{2}\left(1-\lambda t^{2}\right)-\lambda t^{6}\right)}{\left(1-t^{2}\right)^{3}\left(1-\lambda^{2} t^{6}\right)^{2}} \\
& a_{5}=\frac{2 t^{2}\left(1-\lambda^{2} t^{4}\left(1+t^{4}\left(1-\lambda^{2} t^{4}\right)\right)\right)}{\left(1-t^{2}\right)^{2}\left(1-\lambda^{2} t^{6}\right)^{2}} \\
& a_{6}=\frac{-4 t^{2}\left(1+t^{2}\right)\left(1-\lambda t^{2}\left(1+t^{2}\left(1-\lambda t^{2}\right)\right)\right)}{\left(1-t^{2}\right)^{3}\left(1-\lambda^{2} t^{6}\right)} .
\end{aligned}
$$


Axion/dilaton. The axion/dilaton matrix $m_{\alpha \beta}$ is given by

$$
m_{\alpha \beta}=\frac{1}{\xi} m_{a b}=\frac{1}{\xi}\left(\begin{array}{ll}
m_{11} & m_{12} \\
m_{12} & m_{12}
\end{array}\right)
$$

with the components

$$
\begin{aligned}
m_{11} & =\frac{\left(1+\lambda t^{4}\right)}{\left(1-t^{2}\right)^{2}\left(1-\lambda^{2} t^{6}\right)}\left[\left(1-\lambda t^{6}\right)+t^{2}\left(2 w_{1}+1\right)-\lambda t^{4}\left(2 w_{2}+1\right)\right] \\
m_{22} & =\frac{\left(1+\lambda t^{4}\right)}{\left(1-t^{2}\right)^{2}\left(1-\lambda^{2} t^{6}\right)}\left[\left(1-\lambda t^{6}\right)+t^{2}\left(2 w_{1}-1\right)-\lambda t^{4}\left(2 w_{2}-1\right)\right] \\
m_{12} & =\frac{2 w_{2} t^{2}\left(1-\lambda t^{4}\right)\left(1+\lambda t^{2}\right)}{\left(1-t^{2}\right)^{2}\left(1-\lambda^{2} t^{6}\right)}
\end{aligned}
$$

2-form potential. The 2-form potential is given by

$$
C_{\alpha}=C_{m n \alpha} d y^{m} \wedge d y^{n}
$$

The new basis for the 2-forms will be given by the following 6 two-forms

$$
\left\{d w_{1}, d w_{2}, \sigma_{1}\right\} \wedge\left\{\sigma_{2}, \sigma_{3}\right\}
$$

The expression for $C_{\alpha}$ in terms of this basis is rather complicated, but reduces to a manageable expression in the $t \rightarrow 1$ and $\lambda<1$ or $t \rightarrow 1$ limits, which we reported earlier in section 5 .

4-form potential. The 4-form potential is given by

$$
C=\stackrel{\circ}{C}+\frac{1}{4 ! \xi^{2}}\left(f_{1} d_{4}^{1}+f_{2} d_{4}^{2}+f_{3} d_{4}^{3}\right)
$$

with the coefficients

$$
\begin{aligned}
f_{1} & =\frac{-12 w_{2} t^{4}\left(1+\lambda t^{2}\right)^{2}\left(1-\lambda t^{4}\right)^{2}}{\left(1-t^{2}\right)^{4}\left(1-\lambda^{2} t^{6}\right)^{2}} \\
f_{2} & =\frac{12 w_{1} t^{4}\left(1-\lambda t^{2}\right)^{2}\left(1+\lambda t^{4}\right)^{2}}{\left(1-t^{2}\right)^{4}\left(1-\lambda^{2} t^{6}\right)^{2}} \\
f_{3} & =\frac{48 w_{1} w_{2} \lambda t^{6}}{\left(1-t^{2}\right)^{3}\left(1-\lambda^{2} t^{6}\right)}
\end{aligned}
$$


and the 4-forms

$$
\begin{aligned}
d_{4}^{1}= & \epsilon^{i j m} \epsilon^{k l n}\left(u^{m} u^{n}-v^{m} v^{n}\right) d u^{i} \wedge d u^{j} \wedge d v^{k} \wedge d v^{l} \\
= & \frac{\zeta^{1 / 2}}{4} \frac{\left(w_{1}-1\right)}{\left(w_{1}+1\right)} d w_{1} \wedge d w_{2} \wedge \sigma^{2} \wedge \sigma^{3} \\
& +\frac{1}{2}\left(1+w_{1}^{2}-w_{2}^{2}\right) d w_{1} \wedge \sigma^{1} \wedge \sigma^{2} \wedge \sigma^{3}+w_{1} w_{2} d w_{2} \wedge \sigma^{1} \wedge \sigma^{2} \wedge \sigma^{3} \\
d_{4}^{2}= & \epsilon^{i j m} \epsilon^{k l n}\left(u^{m} v^{n}+v^{m} u^{n}\right) d u^{i} \wedge d u^{j} \wedge d v^{k} \wedge d v^{l} \\
= & \frac{\zeta^{1 / 2}}{4} \frac{w_{2}}{\left(w_{1}+1\right)} d w_{1} \wedge d w_{2} \wedge \sigma^{2} \wedge \sigma^{3} \\
& +w_{1} w_{2} d w_{1} \wedge \sigma^{1} \wedge \sigma^{2} \wedge \sigma^{3}+\frac{1}{2}\left(1-w_{1}^{2}+w_{2}^{2}\right) d w_{2} \wedge \sigma^{1} \wedge \sigma^{2} \wedge \sigma^{3} \\
d_{4}^{3}= & d u^{i} \wedge d u^{j} \wedge d v^{i} \wedge d v^{j} \\
= & \frac{\zeta^{1 / 2}}{4} \frac{1}{w_{1}+1} d w_{1} \wedge d w_{2} \wedge \sigma^{2} \wedge \sigma^{3} \\
& +\frac{w_{1}}{2} d w_{1} \wedge \sigma^{1} \wedge \sigma^{2} \wedge \sigma^{3}+\frac{w_{2}}{2} d w_{2} \wedge \sigma^{1} \wedge \sigma^{2} \wedge \sigma^{3}
\end{aligned}
$$

The combined 4-form reads

$$
\begin{aligned}
C=\dot{C}+\frac{t^{4}}{4 \xi^{2}\left(1-t^{2}\right)^{4}\left(1-\lambda^{2} t^{6}\right)^{2}}[ & \tilde{f}_{1} d w_{1} \wedge d w_{2} \wedge \sigma^{2} \wedge \sigma^{3} \\
& +\tilde{f}_{2} d w_{1} \wedge \sigma^{1} \wedge \sigma^{2} \wedge \sigma^{3} \\
& \left.+\tilde{f}_{3} d w_{2} \wedge \sigma^{1} \wedge \sigma^{2} \wedge \sigma^{3}\right]
\end{aligned}
$$

with

$$
\begin{aligned}
& \tilde{f}_{1}=\frac{\zeta^{1 / 2}}{2} \frac{w_{2}}{1+w_{1}}\left(1+\lambda t^{2}\right)^{2}\left(1-\lambda t^{4}\right)^{2} \\
& \tilde{f}_{2}=-w_{2}\left[\left(1+\lambda t^{2}\right)^{2}\left(1-\lambda t^{4}\right)^{2}-w_{1}^{2}\left(1-\lambda t^{2}\right)^{2}\left(1+\lambda t^{4}\right)^{2}-w_{2}^{2}\left(1+\lambda t^{2}\right)^{2}\left(1-\lambda t^{4}\right)^{2}\right] \\
& \tilde{f}_{3}=w_{1}\left[\left(1-\lambda t^{2}\right)^{2}\left(1+\lambda t^{4}\right)^{2}-w_{1}^{2}\left(1-\lambda t^{2}\right)^{2}\left(1+\lambda t^{4}\right)^{2}-w_{2}^{2}\left(1+\lambda t^{2}\right)^{2}\left(1-\lambda t^{4}\right)^{2}\right] .
\end{aligned}
$$

6-form potential. The non-vanishing components of the 6 -form potential are the following

$$
\begin{aligned}
C_{\mu \nu \rho \sigma \tau m}{ }^{\alpha} & =\omega_{\mu \nu \rho \sigma \tau} \Xi_{m}{ }^{\alpha}, \\
C_{\mu \nu \rho \sigma, m n}{ }^{\alpha} & =\omega_{\mu \nu \rho \sigma \tau} g^{\tau \lambda} \Xi_{\lambda m n}{ }^{\alpha},
\end{aligned}
$$

We can transform the one-forms $\Xi^{\alpha}$ and two-forms $\Xi_{\lambda}^{\alpha}$ into the Pilch-Warner basis and rewrite some of the differentials in terms of vector harmonics. The result for the one-forms is

$$
\Xi^{\alpha}=\frac{\sqrt{3}}{2} \frac{\left(\lambda t^{3}+t\right)\left(\lambda^{3} t^{12}+\lambda^{3} t^{10}+\lambda^{2} t^{8}-3 \lambda(\lambda+1) t^{6}+\lambda t^{4}+t^{2}+1\right)}{\left(t^{2}-1\right)^{2}\left(\lambda^{2} t^{6}-1\right)^{2}}\left(\begin{array}{c}
Y_{v}^{(1,1)} \\
-Y_{v}^{(1,0)}
\end{array}\right) .
$$


For the two-forms we get

$$
\begin{aligned}
\Xi_{y}^{\alpha}= & \frac{\sqrt{3}}{\zeta} \frac{t\left(\lambda t^{2}-1\right)\left(\lambda t^{4}+1\right)}{\left(t^{2}-1\right)\left(\lambda^{2} t^{6}-1\right)}\left(\begin{array}{c}
\left(1+w_{1}\right) \sqrt{\zeta} \sigma^{1} \\
-\frac{w_{1} w_{2}}{1+w_{1}} d w_{1}-\left(1-w_{1}\right) d w_{2}+\left(1-w_{1}\right) \sqrt{\zeta} \sigma^{1}
\end{array}\right) \wedge\left(\begin{array}{l}
Y_{v}^{(1,0)} \\
Y_{v}^{(1,1)}
\end{array}\right)(\mathrm{C} .45) \\
& +\frac{\sqrt{3}}{\zeta} \frac{t\left(\lambda^{2} t^{6}+3 \lambda t^{4}-3 \lambda t^{2}-1\right)}{\left(t^{2}-1\right)\left(\lambda^{2} t^{6}-1\right)}\left(\begin{array}{c}
-\frac{1}{2} \frac{\zeta}{1+w_{1}} d w_{1}+w_{2} \sqrt{\zeta} \sigma^{1}-w_{1} d w_{1}-w_{2} d w_{2} \\
-\frac{1}{2} \frac{\zeta}{1+w_{1}} d w_{1}+w_{2} \sqrt{\zeta} \sigma^{1}
\end{array}\right) \wedge\left(\begin{array}{c}
Y_{v}^{(1,1)} \\
Y_{v}^{(1,0)}
\end{array}\right)
\end{aligned}
$$

Note that there is further $t$-dependence in the volume form $\omega_{\mu \nu \rho \sigma \tau}$ and the inverse metric $g^{\tau \lambda}$. In the basis where $t$ is used for the radial coordinate they are given by

$$
\begin{aligned}
g^{\lambda \tau} & =\operatorname{diag}\left(-e^{-2 \phi(t)}, e^{-2 \phi(t)}, e^{-2 \phi(t)}, e^{-2 \phi(t)}, t^{2}\right) \\
e^{2 \phi(t)} & =\frac{1}{t^{2}}\left(1-t^{2}\right)\left(1-\lambda^{2} t^{6}\right)^{1 / 3} e^{2 C_{1}} \\
\omega_{\mu \nu \rho \sigma \tau} & =\frac{1}{t} e^{4 \phi(t)} \epsilon_{\mu \nu \rho \sigma \tau}=\frac{1}{t^{5}}\left(1-t^{2}\right)^{2}\left(1-\lambda^{2} t^{6}\right)^{2 / 3} e^{4 C_{1}} \epsilon_{\mu \nu \rho \sigma \tau} .
\end{aligned}
$$

\section{D $\quad S^{5}$ spherical harmonics with $\mathrm{SO}(3)_{\text {diag }}$ symmetry}

In this appendix we would like to list a subset of the $S^{5}$ scalar, vector, and tensor spherical harmonics that are invariant under the $\mathrm{SO}(3)_{\text {diag }} \subset \mathrm{SO}(3) \times \mathrm{SO}(3) \subset \mathrm{SO}(6)$ symmetry. These harmonics can be found by solving the following defining Laplace eigenvalue equations under the constraint that the solutions be $\mathrm{SO}(3)_{\text {diag-invariant: }}$

$$
\begin{aligned}
& 0=\stackrel{\circ}{\nabla}^{2} Y^{(k, m)}+k(k+4) Y^{(k, m)} \\
& 0=\stackrel{\circ}{\nabla}^{2} Y_{v n}^{(k, m)}+\left(k^{2}+6 k+4\right) Y_{v n}^{(k, m)} \\
& 0=\stackrel{\circ}{\nabla}^{2} Y_{t[n p]}^{(k, m)}+\left(k^{2}+6 k+3\right) Y_{t[n p]}^{(k, m)} \\
& 0=\stackrel{\circ}{\nabla}^{n} Y_{v n}^{(k, m)}=\stackrel{\circ}{\nabla}^{n} Y_{t[n p]}^{(k, m)} \\
& k=0,1,2, \ldots \text {. }
\end{aligned}
$$

The scalar harmonics are denoted by $Y^{(k, m)}$, the vector harmonics by $Y_{v n}^{(k, m)}$, and the tensor harmonics by $Y_{t[n]]}^{(k, m)}$. The symbol $\stackrel{\circ}{\nabla}$ is the covariant derivative on the $S^{5}$, the indices $n, p \in\{1, \ldots, 5\}$ in $Y_{v n}^{(k, m)}$ and $Y_{t[n p]}^{(k, m)}$ refer to the $S^{5}$ cotangent space, and the indices ' $v$ ' and ' $t$ ' stand for "vector" and "tensor". The integer $m$ measures the degeneracy of the harmonics for a given $k$. For scalar harmonics $m$ appears in the eigenvalue of the following differential operator:

$$
\frac{1}{\sin \phi} \frac{\partial}{\partial \phi}\left(\sin \phi \frac{\partial}{\partial \phi} Y^{(k, m)}\right)+m(m+1) Y^{(k, m)}=0 .
$$

In what follows we will normalize the harmonics so that

$$
\begin{aligned}
\int_{S^{5}} Y^{(k, m)} Y^{\left(k^{\prime}, m^{\prime}\right)} & =\int_{S^{5}} Y_{v n}^{(k, m)} Y_{v}^{n\left(k^{\prime}, m^{\prime}\right)}=\int_{S^{5}} Y_{t[n p]}^{(k, m)} Y_{t}^{[n p]\left(k^{\prime}, m^{\prime}\right)} \\
& =\frac{\pi^{3}}{2^{k-1}(k+1)(k+2)} \delta^{k k^{\prime}} \delta^{m m^{\prime}} .
\end{aligned}
$$


The lowest scalar harmonics are given by

$$
\begin{array}{rlrl}
Y^{(0,0)} & =1 & & =\frac{1}{\sqrt{6}}\left(u^{2}-v^{2}\right) \\
Y^{(2,0)} & =\frac{1}{\sqrt{6}} \cos 2 \theta & & \sqrt{\frac{2}{3}}(u \cdot v) \\
Y^{(2,1)} & =\frac{1}{\sqrt{6}} \cos \phi \sin 2 \theta & & \frac{1}{4 \sqrt{15}}\left(3 u^{4}-10 u^{2} v^{2}+3 v^{4}\right) \\
Y^{(4,0)} & =\frac{1}{4 \sqrt{15}}(2 \cos 4 \theta+1) & & \sqrt{\frac{2}{5}}(u \cdot v)\left(u^{2}-v^{2}\right) \\
Y^{(4,1)} & =\frac{1}{2 \sqrt{10}} \cos \phi \sin 4 \theta & & \sqrt{\frac{2}{15}}\left[3(u \cdot v)^{2}-u^{2} v^{2}\right] \\
Y^{(4,2)} & =\frac{1}{\sqrt{30}} \sin ^{2} \theta \cos ^{2} \theta(3 \cos 2 \phi+1) & & \frac{1}{4 \sqrt{7}}\left(u^{2}-v^{2}\right)\left(u^{4}-6 u^{2} v^{2}+v^{4}\right) \\
Y^{(6,0)} & =\frac{1}{8 \sqrt{7}}(\cos 2 \theta+\cos 6 \theta) & & \frac{1}{2 \sqrt{35}}(u \cdot v)\left(5 u^{4}-14 u^{2} v^{2}+5 v^{4}\right) \\
Y^{(6,1)} & =\frac{1}{8 \sqrt{35}} \cos \phi(\sin 2 \theta+3 \sin 6 \theta) & & \frac{1}{\sqrt{7}}\left(u^{2}-v^{2}\right)\left[3(u \cdot v)^{2}-u^{2} v^{2}\right] \\
Y^{(6,2)} & =\frac{1}{2 \sqrt{7}} \sin ^{2} \theta \cos ^{2} \theta \cos 2 \theta(3 \cos 2 \phi+1) \\
Y^{(6,3)} & =\frac{1}{\sqrt{35}} \sin ^{3} \theta \cos ^{3} \theta \cos \phi(5 \cos 2 \phi-1) & =\frac{2}{\sqrt{35}}(u \cdot v)\left[5(u \cdot v)^{2}-3 u^{2} v^{2}\right] .
\end{array}
$$

The transformation from the angle coordinates $(\theta, \phi)$ to the embedding coordinates $(\vec{u}, \vec{v})$ is performed using $\cos ^{2} \theta=u^{2}, \sin ^{2} \theta=v^{2}$, and $\sin \theta \cos \theta \cos \phi=u \cdot v$. The scalar harmonics can also be converted to the $\left(w_{1}, w_{2}\right)$ basis using $w_{1}=2 u^{2}-1=1-2 v^{2}=u^{2}-v^{2}$ and $w_{2}=2 u \cdot v$. One obtains the following expressions

$$
\begin{aligned}
& Y^{(0,0)}=1 \\
& Y^{(2,0)}=\frac{w_{1}}{\sqrt{6}} \\
& Y^{(2,1)}=\frac{w_{2}}{\sqrt{6}} \\
& Y^{(4,0)}=\frac{4 w_{1}^{2}-1}{4 \sqrt{15}} \\
& Y^{(4,1)}=\frac{w_{1} w_{2}}{\sqrt{10}} \\
& Y^{(4,2)}=\frac{w_{1}^{2}+3 w_{2}^{2}-1}{2 \sqrt{30}} \\
& Y^{(6,0)}=\frac{w_{1}\left(2 w_{1}^{2}-1\right)}{4 \sqrt{7}} \\
& Y^{(6,1)}=\frac{w_{2}\left(6 w_{1}^{2}-1\right)}{4 \sqrt{35}}
\end{aligned}
$$




$$
\begin{aligned}
& Y^{(6,2)}=\frac{w_{1}\left(w_{1}^{2}+3 w_{2}^{2}-1\right)}{4 \sqrt{7}} \\
& Y^{(6,3)}=\frac{w_{2}\left(3 w_{1}+5 w_{2}^{2}-3\right)}{4 \sqrt{35}}
\end{aligned}
$$

The lowest vector harmonics that are used in the text are given by

$$
\begin{aligned}
& Y_{v}^{(0,0)}=\cos \theta \sin \theta \sin \phi\left(2 \sigma^{1}+d \phi\right)-\cos \phi d \theta \quad=u^{i} d v^{i}-v^{i} d u^{i} \\
& Y_{v}^{(1,0)}=\frac{2}{\sqrt{3}} \sin \theta \cos ^{2} \theta \sin \phi \sigma^{2} \quad=\frac{2}{\sqrt{3}}\left(\epsilon^{i j k} v^{i} u^{j} d u^{k}\right) \\
& Y_{v}^{(1,1)}=\frac{2}{\sqrt{3}} \sin ^{2} \theta \cos \theta \sin \phi\left(\cos \phi \sigma^{2}-\sin \phi \sigma^{3}\right) \quad=\frac{2}{\sqrt{3}}\left(\epsilon^{i j k} v^{i} u^{j} d v^{k}\right) .
\end{aligned}
$$

We can change the $(\theta, \phi)$ coordinates to $\left(w_{1}, w_{2}\right)$ and re-write the harmonics as follows

$$
\begin{aligned}
Y_{v}^{(0,0)} & =\sqrt{\zeta} \sigma^{1}+\frac{1}{4}\left(\frac{1+w_{1}}{2}\right)^{-1} w_{2} d w_{1}-\frac{1}{2} d w_{2} \\
Y_{v}^{(1,0)} & =\sqrt{\frac{1}{3}}\left(\frac{1+w_{1}}{2}\right)^{1 / 2} \sqrt{\zeta} \sigma^{2} \\
Y_{v}^{(1,1)} & =\sqrt{\frac{1}{12}}\left(\frac{1+w_{1}}{2}\right)^{-1 / 2}\left(w_{2} \sqrt{\zeta} \sigma^{2}-\zeta \sigma^{3}\right) .
\end{aligned}
$$

Note that we view the vector harmonics as one-forms $Y_{v}^{(k, m)}$ on the $S^{5}$ cotangent space. Their components $Y_{v n}^{(k, m)}$ can be read off by choosing a basis of one-forms and expanding the vector harmonics in that basis.

The lowest tensor harmonics corresponding to $k=0$ are given by the following twoforms on the $S^{5}$ cotangent space:

$$
\begin{aligned}
Y_{t}^{(0,0)} & =\epsilon^{i j k}\left(u^{i} d u^{j} \wedge d u^{k}\right) \\
Y_{t}^{(0,1)} & =\frac{1}{\sqrt{3}} \epsilon^{i j k}\left(v^{i} d u^{j} \wedge d u^{k}+2 u^{i} d u^{j} \wedge d v^{k}\right) \\
Y_{t}^{(0,2)} & =\frac{1}{\sqrt{3}} \epsilon^{i j k}\left(u^{i} d v^{j} \wedge d v^{k}+2 v^{i} d v^{j} \wedge d u^{k}\right) \\
Y_{t}^{(0,3)} & =\epsilon^{i j k}\left(v^{i} d v^{j} \wedge d v^{k}\right) .
\end{aligned}
$$

We can change to the $\left(w_{1}, w_{2}\right)$ basis as before and write the tensor harmonics in terms of the vector harmonics as follows:

$$
\begin{aligned}
& Y_{t}^{(0,0)}=\frac{\sqrt{3}}{\zeta}\left(1+w_{1}\right)\left(Y_{v}^{(0,0)}-\frac{w_{2} d w_{1}}{2\left(1+w_{1}\right)}+\frac{d w_{2}}{2}\right) \wedge Y_{v}^{(1,0)} \\
& Y_{t}^{(0,1)}=\frac{1}{\zeta}\left(1+w_{1}\right)\left(Y_{v}^{(0,0)}-\frac{w_{2} d w_{1}}{2\left(1+w_{1}\right)}+\frac{d w_{2}}{2}\right) \wedge Y_{v}^{(1,1)}+\frac{1}{\zeta}\left(2 w_{2} Y_{v}^{(0,0)}-d w_{1}\right) \wedge Y_{v}^{(1,0)} \\
& Y_{t}^{(0,2)}=\frac{1}{\zeta}\left(1-w_{1}\right)\left(Y_{v}^{(0,0)}-\frac{w_{2} d w_{1}}{2\left(1-w_{1}\right)}-\frac{d w_{2}}{2}\right) \wedge Y_{v}^{(1,0)}+\frac{1}{\zeta}\left(2 w_{2} Y_{v}^{(0,0)}-d w_{1}\right) \wedge Y_{v}^{(1,1)} \\
& Y_{t}^{(0,3)}=\frac{\sqrt{3}}{\zeta}\left(1-w_{1}\right)\left(Y_{v}^{(0,0)}-\frac{w_{2} d w_{1}}{2\left(1-w_{1}\right)}-\frac{d w_{2}}{2}\right) \wedge Y_{v}^{(1,1)}
\end{aligned}
$$




\section{E UV asymptotics of the GPPZ uplift}

In this appendix we collect the UV expansion of the various fields in our solution. Contrary to main text, we keep the explicit dependence on the angles $\varphi$ and $\omega$ in (3.6). When possible we express the results on the basis of $S^{5}$ harmonics of appendix D.

Dilaton/axion. The expansion of the axio-dilaton field up to terms of order $1 / r^{4}$ is

$$
\begin{aligned}
B= & \frac{1+i \tau}{1-i \tau} \sim-\frac{m_{0}^{2}}{r^{2}} \frac{4 \sqrt{2}}{3 \sqrt{3}} e^{-2 i \varphi}\left(Y^{(2,0)}+i Y^{(2,1)}\right) \\
& +\frac{1}{r^{4}}\left[\frac{2 \sqrt{2}}{3} m_{0} \sigma_{0} e^{-i(\varphi-\omega)}\left(Y^{(2,0)}-i Y^{(2,1)}\right)+\frac{4 \sqrt{2}}{9 \sqrt{3}} m_{0}^{4} e^{-2 i \varphi}\left(Y^{(2,0)}+i Y^{(2,1)}\right)\right]
\end{aligned}
$$

with $m_{0}$ and $\sigma_{0}$ given in (2.14). The first terms of the expansions of the dilaton and axion are

$$
\begin{aligned}
e^{\Phi} & =1+\frac{m_{0}^{2}}{r^{2}} A_{1}+\frac{1}{r^{4}}\left(m_{0} \sigma_{0} A_{2}+m_{0}^{4} A_{3}\right) \\
C_{0} & =\frac{m_{0}^{2}}{r^{2}} B_{1}+\frac{1}{r^{4}}\left(m_{0} \sigma_{0} B_{2}+m_{0}^{4} B_{3}\right)
\end{aligned}
$$

where the coefficients $A_{i}$ and $B_{i}$ can be expressed in terms of scalar harmonics as

$$
\begin{aligned}
A_{1}= & -\frac{2 \sqrt{2}}{\sqrt{3}}\left(\sin 2 \varphi Y^{(2,1)}+\cos 2 \varphi Y^{(2,0)}\right) \\
B_{1}= & \frac{2 \sqrt{2}}{\sqrt{3}}\left(\cos 2 \varphi Y^{(2,1)}-\sin 2 \varphi Y^{(2,0)}\right) \\
A_{2}= & \sqrt{2}\left(\cos (\varphi-\omega) Y^{(2,0)}-\sin (\varphi-\omega) Y^{(2,1)}\right) \\
B_{2}= & \sqrt{2}\left(\sin (\varphi-\omega) Y^{(2,0)}+\cos (\varphi-\omega) Y^{(2,1)}\right) \\
A_{3}= & \frac{1}{9} Y^{(0,0)}+\frac{2 \sqrt{2}}{3 \sqrt{3}}\left(\sin 2 \varphi Y^{(2,1)}-\cos 2 \varphi Y^{(2,0)}\right)+\frac{4 \sqrt{5}}{9 \sqrt{3}}\left(Y^{(4,0)}-\sqrt{2} Y^{(4,2)}\right) \\
B_{3}= & -\frac{2 \sqrt{2}}{3 \sqrt{3}}\left(\sin 2 \varphi Y^{(2,0)}+\cos 2 \varphi Y^{(2,1)}\right) \\
& -\frac{4 \sqrt{10}}{9 \sqrt{3}}\left(\sqrt{2} \sin 4 \varphi Y^{(4,0)}-\sqrt{3} \cos 4 \varphi Y^{(4,1)}-\sin 4 \varphi Y^{(4,2)}\right)
\end{aligned}
$$

Metric. The large $r$ behaviour of the internal five-dimensional metric is, up to order $1 / r^{4}$

$$
\begin{aligned}
d s_{5}^{2}= & \left(d u_{i}\right)^{2}+\left(d v_{i}\right)^{2}+\frac{1}{r^{2}}\left(A_{i j} d u_{i} d u_{j}+C_{i j} d v_{i} d v_{j}+E_{i j} d u_{i} d v_{j}\right) \\
& +\frac{1}{r^{4}}\left(B_{i j} d u_{i} d u_{j}+D_{i j} d v_{i} d v_{j}+F_{i j} d u_{i} d v_{j}\right)
\end{aligned}
$$


with

$$
\begin{aligned}
& A_{i j}=-\frac{m_{0}^{2}}{6}\left(3+4\left(u^{2}-v^{2}\right)\right) \delta_{i j}+\frac{m_{0}^{2}}{3} v_{i} v_{j} \\
& B_{i j}= \frac{m_{0}^{4}}{72}\left(7+40\left(u^{2}-v^{2}\right)+96(u \cdot v)^{2}+24\left(u^{2}-v^{2}\right)^{2}\right) \\
&\left.+\frac{1}{\sqrt{3}} m_{0} \sigma_{0}\left(\cos (\varphi+\omega)\left(u^{2}-v^{2}\right)+2 \sin (\varphi+\omega)(u \cdot v)\right)\right] \delta_{i j} \\
&+\frac{1}{\sqrt{3}} m_{0} \sigma_{0}[1-\cos (\varphi+\omega)] u_{i} u_{j}+\left(\frac{m_{0}^{4}}{6}+\frac{1}{\sqrt{3}} \sigma_{0} m_{0} \cos (\varphi+\omega)\right) v_{i} v_{j} \\
&+\frac{1}{\sqrt{3}} m_{0} \sigma_{0} \sin (\varphi+\omega)\left(u_{i} v_{j}+v_{i} u_{j}\right) \\
& C_{i j}=-\frac{m_{0}^{2}}{6}\left(3-4\left(u^{2}-v^{2}\right)\right) \delta_{i j}+\frac{m_{0}^{2}}{3} u_{i} u_{j} \\
& D_{i j}= \frac{m_{0}^{4}}{72}\left(7-40\left(u^{2}-v^{2}\right)+96(u \cdot v)^{2}+24\left(u^{2}-v^{2}\right)^{2}\right) \\
&\left.-\frac{1}{\sqrt{3}} m_{0} \sigma_{0}\left(\cos (\varphi+\omega)\left(u^{2}-v^{2}\right)+2 \sin (\varphi+\omega)(u \cdot v)\right)\right] \delta_{i j} \\
&+\frac{1}{\sqrt{3}} m_{0} \sigma_{0}[1-\cos (\varphi+\omega)] v_{i} v_{j}+\left(\frac{m_{0}^{4}}{6}+\frac{1}{\sqrt{3}} \sigma_{0} m_{0} \cos (\varphi+\omega)\right) u_{i} u_{j} \\
&-\frac{1}{\sqrt{3}} m_{0} \sigma_{0} \sin (\varphi+\omega)\left(u_{i} v_{j}+v_{i} u_{j}\right) \\
& E_{i j}= \frac{m_{0}^{2}}{3}\left(-8(u \cdot v) \delta_{i j}-8 u_{i} v_{j}+6 v_{i} u_{j}\right) \\
& F_{i j}=\left.\frac{20 m_{0}^{4}}{9}(u \cdot v)+\frac{2}{\sqrt{3}} m_{0} \sigma_{0}\left(\sin (\varphi+\omega)\left(u^{2}-v^{2}\right)-2 \cos (\varphi+\omega)(u \cdot v)\right)\right] \delta_{i j} \\
&+\frac{4 m_{0}^{4}}{9}\left[1+\frac{3 \sqrt{3} \sigma_{0}}{2 m_{0}^{3}}\right] m_{0} \sigma_{0} \sin (\varphi+\omega)\left(u_{i} v_{j}+\frac{m_{0}^{4}}{9}\left[-7+\frac{6 \sqrt{3} \sigma_{0}}{m_{0}^{3}}\right] v_{i} u_{j} v_{j}\right) \\
&(
\end{aligned}
$$

Two-form potentials. For generic values of the angles $\varphi$ and $\omega$, the two-form potentials are

$$
\begin{aligned}
C_{1}= & -\frac{1}{2} \epsilon_{i j k}\left[\frac{a_{-}(u, v)}{\sqrt{3}} \frac{m_{0}}{r}-\frac{d_{-}(v, u)}{2} \frac{\sigma_{0}}{r^{3}}\right. \\
& \left.-\frac{\left.2 c_{+}(v, u)+2\left(3 f_{-}^{1} u_{i}+f_{-}^{2} v_{i}\right)\left(u^{2}-v^{2}\right)-4\left[3 f_{+}^{2} u_{i}-f_{+}^{1} v_{i}\right](u \cdot v)\right)}{3 \sqrt{3}} \frac{m_{0}^{3}}{r^{3}}\right] d u_{j} \wedge d u_{k} \\
& -\frac{1}{2} \epsilon_{i j k}\left[-\frac{b_{-}(v, u)}{\sqrt{3}} \frac{m_{0}}{r}+\frac{d_{-}(v, u)}{2} \frac{\sigma_{0}}{r^{3}}\right. \\
& \left.+\frac{2 c_{-}(v, u)-2\left(f_{-}^{1} u_{i}+3 f_{-}^{2} v_{i}\right)\left(u^{2}-v^{2}\right)+4\left(f_{+}^{2} u_{i}-3 f_{+}^{1} v_{i}\right)(u \cdot v)}{3 \sqrt{3}} \frac{m_{0}^{3}}{r^{3}}\right] d v_{j} \wedge d v_{k}
\end{aligned}
$$




$$
\begin{aligned}
+ & \epsilon_{i j k}\left[\frac{c_{-}(u, v)}{\sqrt{3}} \frac{m_{0}}{r}-\frac{d_{-}(u, v)}{2} \frac{\sigma_{0}}{r^{3}}\right. \\
& \left.-\frac{2 c_{-}(u, v)-2\left(f_{-}^{2} u_{i}+f_{-}^{1} v_{i}\right)\left(u^{2}-v^{2}\right)-4\left(f_{+}^{1} u_{i}-f_{+}^{2} v_{i}\right)(u \cdot v)}{3 \sqrt{3}} \frac{m_{0}^{3}}{r^{3}}\right] d u_{j} \wedge d v_{k} \\
C_{2}= & -\frac{1}{2} \epsilon_{i j k}\left[\frac{b_{+}(u, v)}{\sqrt{3}} \frac{m_{0}}{r}-\frac{d_{+}(u, v)}{2} \frac{\sigma_{0}}{r^{3}}\right. \\
& \left.-\frac{2 c_{-}(u, v)-2\left(3 g_{+}^{1} u_{i}+g_{+}^{2} v_{i}\right)\left(u^{2}-v^{2}\right)-4\left(3 g_{-}^{2} u_{i}-g_{-}^{1} v_{i}\right)(u \cdot v)}{3 \sqrt{3}} \frac{m_{0}^{3}}{r^{3}}\right] d u_{j} \wedge d u_{k} \\
& -\frac{1}{2} \epsilon_{i j k}\left[\frac{a_{+}(v, u)}{\sqrt{3}} \frac{m_{0}}{r}+\frac{d_{+}(u, v)}{2} \frac{\sigma_{0}}{r^{3}}\right. \\
& \left.+\frac{2 c_{-}(u, v)+2\left(g_{+}^{1} u_{i}+3 g_{+}^{2} v_{i}\right)\left(u^{2}-v^{2}\right)+4\left(g_{-}^{2} u_{i}-3 g_{-}^{1} v_{i}\right)(u \cdot v)}{3 \sqrt{3}} \frac{m_{0}^{3}}{r^{3}}\right] d v_{j} \wedge d v_{k} \\
& -\epsilon_{i j k}\left[\frac{c_{+}(v, u)}{\sqrt{3}} \frac{m_{0}}{r}+\frac{d_{-}(v, u)}{2} \frac{\sigma_{0}}{r^{3}}\right. \\
& \left.-\frac{2 c_{+}(v, u)-2\left(g_{+}^{2} u_{i}+g_{+}^{1} v_{i}\right]\left(u^{2}-v^{2}\right)+4\left(g_{-}^{1} u_{i}-g_{-}^{2} v_{i}\right)(u \cdot v)}{3 \sqrt{3}} \frac{m_{0}^{3}}{r^{3}}\right] d u_{j} \wedge d v_{k}
\end{aligned}
$$

where

$$
\begin{array}{ll}
a^{ \pm}(x, y)=3 \sin \varphi x_{i} \pm \cos \varphi y_{i} & c^{ \pm}(x, y)=\cos \varphi x_{i} \pm \sin \varphi y_{i} \\
b^{ \pm}(x, y)=3 \cos \varphi x_{i} \pm \sin \varphi y_{i} & d^{ \pm}(x, y)=\cos \omega x_{i} \pm \sin \omega y_{i}
\end{array}
$$

and

$$
\begin{aligned}
& f_{ \pm}^{1}=\sin \frac{\varphi+\omega}{4} \cos \frac{3 \varphi-\omega}{4} \pm \cos 3 \frac{\varphi+\omega}{4} \sin \frac{3 \varphi-\omega}{4} \\
& f_{ \pm}^{2}=\cos \frac{\varphi+\omega}{4} \cos \frac{3 \varphi-\omega}{4} \pm \sin 3 \frac{\varphi+\omega}{4} \sin \frac{3 \varphi-\omega}{4} \\
& g_{ \pm}^{1}=\cos 3 \frac{\varphi+\omega}{4} \cos \frac{3 \varphi-\omega}{4} \pm \sin \frac{\varphi+\omega}{4} \sin \frac{3 \varphi-\omega}{4} \\
& g_{ \pm}^{2}=\sin 3 \frac{\varphi+\omega}{4} \cos \frac{3 \varphi-\omega}{4} \pm \cos \frac{\varphi+\omega}{4} \sin \frac{3 \varphi-\omega}{4}
\end{aligned}
$$

\section{F One field truncations}

\section{F.1 The case $m(y)=0$}

For the truncation $m(y) \equiv 0$ the uplift of the resulting $D=5$ theory has been given by Gubser, Herzog, Pufu and Tesileanu [22], see also [23, 24]. In the language of our uplift this corresponds to setting $\nu \equiv 1$. After applying this to our uplift, and using the usual definition $\mu=e^{\sigma}$ the warp factor $\Delta$ is simply given by

$$
\Delta \rightarrow(\cosh \sigma)^{-3 / 2}
$$


and the IIB metric takes the form

$$
\begin{aligned}
d s_{10}^{2}= & \cosh \sigma\left[(\cosh \sigma)^{-2 / 3} e^{2 y} \eta_{\mu \nu} d x^{\mu} d x^{\nu}+d y^{2}\right] \\
& +\frac{1}{\cosh \sigma}\left[d u^{i} d u^{i}+d v^{i} d v^{i}+\left(\sinh \sigma Y_{v}^{(0,0)}\right)^{2}\right] \\
= & \cosh \sigma\left[\left(\frac{\sigma_{0}}{2 \sinh \sigma}\right)^{2 / 3} \eta_{\mu \nu} d x^{\mu} d x^{\nu}+\left(\frac{2 d \sigma}{3 \sinh (2 \sigma)}\right)^{2}+\frac{d \Omega_{5}^{2}}{\cosh ^{2} \sigma}+\left(\tanh \sigma Y_{v}^{(0,0)}\right)^{2}\right] .
\end{aligned}
$$

In the second line in the metric we change the radial variable from $y$ to $\sigma$. The field $\sigma(y)$ is monotonic in $y$ for $y \in\left\{C_{2}, \infty\right\}$, and is therefore invertible. The range of $\sigma$ is $\sigma \in\{0, \infty\}$, where $\sigma=0$ corresponds to the AdS boundary, and $\sigma=\infty$ to the singularity. The Ricci scalar takes the simple form

$$
R=18 \sinh \sigma \tanh \sigma .
$$

The asymptotic behaviour of the metric for $\sigma \rightarrow \infty$, which is the expansion around the singularity is the following

$$
\begin{aligned}
d s_{10}^{2} & =\left(\frac{1}{2} e^{\sigma}\right)^{1 / 3} \eta_{\mu \nu} d x^{\mu} d x^{\nu}+\frac{1}{9}\left(\frac{1}{2} e^{\sigma}\right)^{-3} d \sigma^{2}+\frac{1}{2} e^{\sigma} d \Omega_{5}^{2}+\frac{1}{2} e^{\sigma}\left(Y_{v}^{(0,0)}\right)^{2}+\ldots \\
& =\rho^{2 / 3} \eta_{\mu \nu} d x^{\mu} d x^{\nu}+\left(\frac{2}{3} \frac{d \rho}{\rho^{4}}\right)^{2}+\rho^{2}\left[d \Omega_{5}^{2}+\left(Y_{v}^{(0,0)}\right)^{2}\right]+\ldots
\end{aligned}
$$

where we defined $\rho^{2}=\frac{1}{2} e^{\sigma}$. The asymptotic behaviour of the metric for $\sigma \rightarrow 0$, which is the expansion around the conformal boundary of the AdS, is the following

$$
\begin{aligned}
d s_{10}^{2} \rightarrow & \left(\frac{\sigma_{0}}{2 \sigma}\right)^{2 / 3} \eta_{\mu \nu} d x^{\mu} d x^{\nu}+\left(\frac{d \sigma}{3 \sigma}\right)^{2}+d \Omega_{5}^{2} \\
& +\sigma^{2}\left[\frac{7}{18}\left(\frac{\sigma_{0}}{2 \sigma}\right)^{2 / 3} \eta_{\mu \nu} d x^{\mu} d x^{\nu}-\frac{5}{54} d \sigma^{2}-\frac{1}{2} d \Omega_{5}^{2}+\left(Y_{v}^{(0,0)}\right)^{2}\right]+\ldots \\
= & \frac{\eta_{\mu \nu} d x^{\mu} d x^{\nu}+d z^{2}}{z^{2}}+d \Omega_{5}^{2} \\
& +\frac{\sigma_{0}^{2}}{4} z^{6}\left[\frac{7}{18} \frac{1}{z^{2}} \eta_{\mu \nu} d x^{\mu} d x^{\nu}-\frac{5}{24} \sigma_{0}^{2} z^{4} d z^{2}-\frac{1}{2} d \Omega_{5}^{2}+\left(Y_{v}^{(0,0)}\right)^{2}\right]+\ldots
\end{aligned}
$$

where we have substituted $z=\left(\frac{\sigma_{0}}{2 \sigma}\right)^{-1 / 3}$.

In this truncation, the dilaton/axion matrix reduces to the identity, and the two form is given by

$$
C_{m n \alpha}=\mathcal{S}_{\alpha}{ }^{a} \mathrm{C}_{m n a}
$$

with

$$
\begin{aligned}
& \mathrm{C}_{1}=-\frac{1}{4}(1-\tanh \sigma) \varepsilon^{i j k}\left(V^{i}\left(\Theta^{j} \wedge \Theta^{k}-\Lambda^{j} \wedge \Lambda^{k}\right)+2 U^{i} \Theta^{j} \wedge \Lambda^{k}\right), \\
& \mathrm{C}_{2}=-\left.\mathrm{C}_{1}\right|_{U^{i} \leftrightarrow V^{i}, \Theta^{i} \leftrightarrow \Lambda^{i}} .
\end{aligned}
$$




\section{F.2 The case $\sigma(y)=0$}

Another consistent truncation to one scalar corresponds to setting $\sigma(y)=0$, i.e. $\mu \equiv 1$. In this case, the IIB metric takes the form

$$
d s_{\mathrm{IIB}}^{2}=\Delta^{-2 / 3}\left(g_{\mu \nu}(x) d x^{\mu} d x^{\nu}+\Delta^{8 / 3} d \hat{s}_{5}^{2}\right),
$$

with the warp factor $\Delta$ and the internal metric $d \hat{s}_{5}^{2}$ given by

$$
\begin{aligned}
\Delta^{-8 / 3}= & \frac{\left(1+\nu^{2}\right)^{3}\left(1+\nu^{6}\right)}{16 \nu^{6}}+\frac{U^{2} V^{2}-(U \cdot V)^{2}}{16 \nu^{8}}\left(1-\nu^{4}\right)^{4} \\
d \hat{s}_{5}^{2}= & \frac{\left(1+\nu^{4}\right)\left(1+\nu^{2}\right)^{2}}{8 \nu^{4}}\left(\Theta^{i} \Theta^{i}+\Lambda^{i} \Lambda^{i}\right) \\
& -\frac{\left(1-\nu^{4}\right)^{2}}{8 \nu^{4}}\left(\left(U^{2}-V^{2}\right)\left(\Theta^{i} \Theta^{i}-\Lambda^{i} \Lambda^{i}\right)+4(U \cdot V) \Theta^{i} \Lambda^{i}\right) \\
& +\frac{\left(1-\nu^{4}\right)\left(1-\nu^{2}\right)\left(1+\nu^{6}\right)}{16 \nu^{6}}\left(\left(V^{i} \Theta^{i}\right)\left(V^{j} \Theta^{j}\right)+\left(U^{i} \Lambda^{i}\right)\left(U^{j} \Lambda^{j}\right)\right) \\
& +\frac{\left(1-\nu^{4}\right)\left(1+\nu^{2}\right)\left(1-\nu^{6}\right)}{8 \nu^{6}}\left(V^{i} \Theta^{i}\right)\left(U^{j} \Lambda^{j}\right) \\
& -\frac{\left(1-\nu^{4}\right)\left(1-\nu^{8}\right)}{4 \nu^{6}}\left(U^{i} \Theta^{i}\right)\left(V^{j} \Lambda^{j}\right),
\end{aligned}
$$

respectively. The dilaton/axion matrix $m_{\alpha \beta}$ is given by

$$
m_{\alpha \beta}=\Delta^{4 / 3} \mathcal{S}_{\alpha}{ }^{a} \mathcal{S}_{\beta}{ }^{b} \mathrm{~m}_{a b},
$$

with the $\mathrm{SO}(2)$ rotation matrix $\mathcal{S}=e^{\frac{3 i}{4} \varphi \sigma_{2}}$ and the matrix $\mathrm{m}_{a b}$ given by

$$
\begin{aligned}
& \mathrm{m}_{11}=\frac{1}{8 \nu^{4}}\left(\left(1+\nu^{4}\right)\left(1+\nu^{2}\right)^{2}+\left(1-\nu^{4}\right)^{2}\left(U^{2}-V^{2}\right)\right), \\
& \mathrm{m}_{12}=\frac{\left(1-\nu^{4}\right)^{2}}{4 \nu^{4}}(U \cdot V), \\
& \mathrm{m}_{22}=\frac{1}{8 \nu^{4}}\left(\left(1+\nu^{4}\right)\left(1+\nu^{2}\right)^{2}-\left(1-\nu^{4}\right)^{2}\left(U^{2}-V^{2}\right)\right) .
\end{aligned}
$$

The two-form doublet is given by

$$
C_{m n \alpha}=\Delta^{8 / 3} \mathcal{S}_{\alpha}{ }^{a} \mathrm{C}_{m n a},
$$

with

$$
\begin{aligned}
& \mathrm{C}_{1}=-\frac{1}{8 \nu^{4}} \mathrm{~m}_{11} \varepsilon^{i j k}\left(\left(1-\nu^{2}\right)\left(1+\nu^{6}\right) V^{i} \Theta^{j} \wedge \Theta^{k}+\left(1+\nu^{2}\right)\left(1-\nu^{6}\right) V^{i} \Lambda^{j} \wedge \Lambda^{k}\right. \\
& \left.+2 \nu^{2}\left(1-\nu^{4}\right) U^{i} \Theta^{j} \wedge \Lambda^{k}\right) \\
& +\frac{1}{8 \nu^{4}} \mathrm{~m}_{12} \varepsilon^{i j k}\left(\left(1+\nu^{2}\right)\left(1-\nu^{6}\right) U^{i} \Theta^{j} \wedge \Theta^{k}+\left(1-\nu^{2}\right)\left(1+\nu^{6}\right) U^{i} \Lambda^{j} \wedge \Lambda^{k}\right. \\
& \left.+2 \nu^{2}\left(1-\nu^{4}\right) V^{i} \Theta^{j} \wedge \Lambda^{k}\right) \\
& \mathrm{C}_{2}=-\left.\mathrm{C}_{1}\right|_{U^{i} \leftrightarrow V^{i}, \Theta^{i} \leftrightarrow \Lambda^{i}} .
\end{aligned}
$$


Finally, the non-vanishing components of the 5-form field strength are given by

$$
\begin{aligned}
F_{\mu \nu \rho \sigma \tau} & =-\frac{1}{3} \omega_{\mu \nu \rho \sigma \tau} V_{\text {pot }}, \\
F_{\mu \nu \rho \sigma m} & =\frac{1}{8} \omega_{\mu \nu \rho \sigma \lambda} \mathcal{J}^{\lambda}\left(U^{i} \Lambda^{i}-V^{i} \Theta^{i}\right)_{m}, \\
F_{\mu \nu \rho m n} & =-\frac{1}{12} \omega_{\mu \nu \rho \sigma \tau} F^{\sigma \tau}\left(\Theta^{i} \wedge \Lambda^{i}\right)_{m n},
\end{aligned}
$$

together with those related by self-duality of the IIB field strength.

Open Access. This article is distributed under the terms of the Creative Commons Attribution License (CC-BY 4.0), which permits any use, distribution and reproduction in any medium, provided the original author(s) and source are credited.

\section{References}

[1] J.M. Maldacena and C. Núñez, Towards the large $N$ limit of pure $N=1$ super Yang-Mills, Phys. Rev. Lett. 86 (2001) 588 [hep-th/0008001] [INSPIRE].

[2] I.R. Klebanov and M.J. Strassler, Supergravity and a confining gauge theory: duality cascades and $\chi S B$ resolution of naked singularities, JHEP 08 (2000) 052 [hep-th/0007191] [INSPIRE].

[3] C. Vafa and E. Witten, A strong coupling test of S duality, Nucl. Phys. B 431 (1994) 3 [hep-th/9408074] [INSPIRE].

[4] R. Donagi and E. Witten, Supersymmetric Yang-Mills theory and integrable systems, Nucl. Phys. B 460 (1996) 299 [hep-th/9510101] [InSPIRE].

[5] J. Polchinski and M.J. Strassler, The string dual of a confining four-dimensional gauge theory, hep-th/0003136 [INSPIRE].

[6] L. Girardello, M. Petrini, M. Porrati and A. Zaffaroni, The supergravity dual of $N=1$ super Yang-Mills theory, Nucl. Phys. B 569 (2000) 451 [hep-th/9909047] [INSPIRE].

[7] M. Pernici, K. Pilch and P. van Nieuwenhuizen, Gauged $N=8 D=5$ supergravity, Nucl. Phys. B 259 (1985) 460 [inSPIRE].

[8] M. Günaydin, L.J. Romans and N.P. Warner, Gauged $N=8$ supergravity in five-dimensions, Phys. Lett. B 154 (1985) 268 [INSPIRE].

[9] A. Baguet, O. Hohm and H. Samtleben, Consistent type IIB reductions to maximal 5D supergravity, Phys. Rev. D 92 (2015) 065004 [arXiv:1506.01385] [INSPIRE].

[10] H.J. Kim, L.J. Romans and P. van Nieuwenhuizen, The mass spectrum of chiral $N=2$ $D=10$ supergravity on $S^{5}$, Phys. Rev. D 32 (1985) 389 [INSPIRE].

[11] O. Aharony, N. Dorey and S.P. Kumar, New modular invariance in the $N=1^{*}$ theory, operator mixings and supergravity singularities, JHEP 06 (2000) 026 [hep-th/0006008] [INSPIRE].

[12] B. Eden, C. Jarczak, E. Sokatchev and Ya. S. Stanev, Operator mixing in $N=4$ SYM: the Konishi anomaly revisited, Nucl. Phys. B 722 (2005) 119 [hep-th/0501077] [INSPIRE].

[13] M. Petrini and A. Zaffaroni, The holographic RG flow to conformal and nonconformal theory, hep-th/0002172 [INSPIRE]. 
[14] M. Bianchi, D.Z. Freedman and K. Skenderis, How to go with an $R G$ flow, JHEP 08 (2001) 041 [hep-th/0105276] [INSPIRE].

[15] M. Bianchi, D.Z. Freedman and K. Skenderis, Holographic renormalization, Nucl. Phys. B 631 (2002) 159 [hep-th/0112119] [INSPIRE].

[16] D. Elander and M. Piai, Light scalars from a compact fifth dimension, JHEP 01 (2011) 026 [arXiv: 1010.1964] [INSPIRE].

[17] O. DeWolfe and D.Z. Freedman, Notes on fluctuations and correlation functions in holographic renormalization group flows, hep-th/0002226 [INSPIRE].

[18] G.W. Gibbons, G.T. Horowitz and P.K. Townsend, Higher dimensional resolution of dilatonic black hole singularities, Class. Quant. Grav. 12 (1995) 297 [hep-th/9410073] [INSPIRE].

[19] D.Z. Freedman, S.S. Gubser, K. Pilch and N.P. Warner, Continuous distributions of D3-branes and gauged supergravity, JHEP 07 (2000) 038 [hep-th/9906194] [INSPIRE].

[20] K. Pilch and N.P. Warner, $N=1$ supersymmetric renormalization group flows from IIB supergravity, Adv. Theor. Math. Phys. 4 (2002) 627 [hep-th/0006066] [INSPIRE].

[21] M. Bianchi, O. DeWolfe, D.Z. Freedman and K. Pilch, Anatomy of two holographic renormalization group flows, JHEP 01 (2001) 021 [hep-th/0009156] [INSPIRE].

[22] S.S. Gubser, C.P. Herzog, S.S. Pufu and T. Tesileanu, Superconductors from superstrings, Phys. Rev. Lett. 103 (2009) 141601 [arXiv:0907.3510] [INSPIRE].

[23] D. Cassani, G. Dall'Agata and A.F. Faedo, Type IIB supergravity on squashed Sasaki-Einstein manifolds, JHEP 05 (2010) 094 [arXiv: 1003.4283] [INSPIRE].

[24] J.P. Gauntlett and O. Varela, Universal Kaluza-Klein reductions of type IIB to $N=4$ supergravity in five dimensions, JHEP 06 (2010) 081 [arXiv: 1003.5642] [INSPIRE].

[25] K.A. Intriligator, Bonus symmetries of $N=4$ super Yang-Mills correlation functions via AdS duality, Nucl. Phys. B 551 (1999) 575 [hep-th/9811047] [INSPIRE].

[26] M. Günaydin and N. Marcus, The spectrum of the $S^{5}$ compactification of the chiral $N=2$, $D=10$ supergravity and the unitary supermultiplets of $\mathrm{U}(2,2 / 4)$, Class. Quant. Grav. 2 (1985) L11 [INSPIRE].

[27] Wolfram Research Inc., Mathematica, version 11.0, U.S.A.

[28] S. de Haro, S.N. Solodukhin and K. Skenderis, Holographic reconstruction of space-time and renormalization in the AdS/CFT correspondence, Commun. Math. Phys. 217 (2001) 595 [hep-th/0002230] [INSPIRE].

[29] K. Skenderis and M. Taylor, Kaluza-Klein holography, JHEP 05 (2006) 057 [hep-th/0603016] [INSPIRE].

[30] R.C. Myers, Dielectric branes, JHEP 12 (1999) 022 [hep-th/9910053] [INSPIRE].

[31] D.Z. Freedman and J.A. Minahan, Finite temperature effects in the supergravity dual of the $N=1^{*}$ gauge theory, JHEP 01 (2001) 036 [hep-th/0007250] [INSPIRE].

[32] S. Schmidt and K. Skenderis, $N=1$ deformations of $N=4$ SYM and SUGRA potentials, to appear.

[33] S.S. Gubser, Curvature singularities: the good, the bad and the naked, Adv. Theor. Math. Phys. 4 (2000) 679 [hep-th/0002160] [INSPIRE]. 
[34] M. Günaydin, L.J. Romans and N.P. Warner, Compact and noncompact gauged supergravity theories in five-dimensions, Nucl. Phys. B 272 (1986) 598 [INSPIRE].

[35] M.J. Duff, G.W. Gibbons and P.K. Townsend, Macroscopic superstrings as interpolating solitons, Phys. Lett. B 332 (1994) 321 [hep-th/9405124] [INSPIRE].

[36] H.J. Boonstra, K. Skenderis and P.K. Townsend, The domain wall/QFT correspondence, JHEP 01 (1999) 003 [hep-th/9807137] [INSPIRE].

[37] I. Kanitscheider, K. Skenderis and M. Taylor, Precision holography for non-conformal branes, JHEP 09 (2008) 094 [arXiv: 0807.3324] [INSPIRE].

[38] R.M. Wald, General relativity, Chicago Univ. Pr., Chicago U.S.A., (1984) [InSPIRE].

[39] K. Pilch and N.P. Warner, A new supersymmetric compactification of chiral IIB supergravity, Phys. Lett. B 487 (2000) 22 [hep-th/0002192] [INSPIRE].

[40] K. Pilch and N.P. Warner, $N=2$ supersymmetric $R G$ flows and the IIB dilaton, Nucl. Phys. B 594 (2001) 209 [hep-th/0004063] [INSPIRE].

[41] A. Brandhuber and K. Sfetsos, An $N=2$ gauge theory and its supergravity dual, Phys. Lett. B 488 (2000) 373 [hep-th/0004148] [INSPIRE].

[42] N. Bobev, F.F. Gautason and J. Van Muiden, Precision holography for $N=2^{*}$ on $S^{4}$ from type IIB supergravity, JHEP 04 (2018) 148 [arXiv:1802.09539] [INSPIRE].

[43] N. Bobev, F.F. Gautason, B.E. Niehoff and J. van Muiden, Uplifting GPPZ: a ten-dimensional dual of $N=1^{*}$, arXiv:1805.03623 [INSPIRE].

[44] A. Khavaev, K. Pilch and N.P. Warner, New vacua of gauged $N=8$ supergravity in five-dimensions, Phys. Lett. B 487 (2000) 14 [hep-th/9812035] [INSPIRE].

[45] H. Nastase and D. Vaman, On the nonlinear KK reductions on spheres of supergravity theories, Nucl. Phys. B 583 (2000) 211 [hep-th/0002028] [INSPIRE].

[46] K. Lee, C. Strickland-Constable and D. Waldram, Spheres, generalised parallelisability and consistent truncations, Fortsch. Phys. 65 (2017) 1700048 [arXiv:1401.3360] [INSPIRE].

[47] F. Ciceri, B. de Wit and O. Varela, IIB supergravity and the $E_{6(6)}$ covariant vector-tensor hierarchy, JHEP 04 (2015) 094 [arXiv: 1412.8297] [INSPIRE]. 